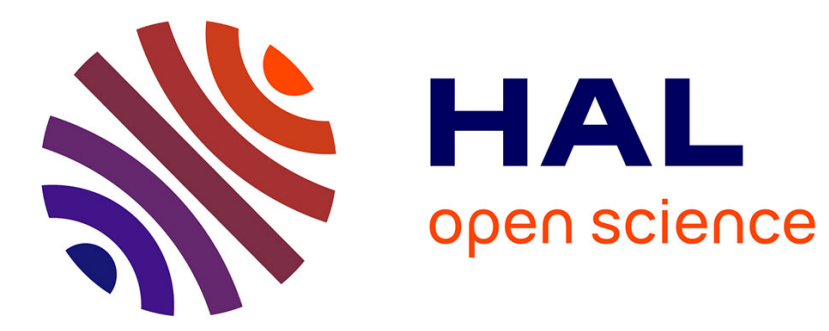

\title{
Three-dimensional stability of vortex arrays in a stratified and rotating fluid
}

Axel Deloncle, Paul Billant, Jean-Marc Chomaz

\section{To cite this version:}

Axel Deloncle, Paul Billant, Jean-Marc Chomaz. Three-dimensional stability of vortex arrays in a stratified and rotating fluid. Journal of Fluid Mechanics, 2011, 678, pp.482-510. 10.1017/jfm.2011.121 . hal-00997999

\section{HAL Id: hal-00997999}

https://hal-polytechnique.archives-ouvertes.fr/hal-00997999

Submitted on 5 Jun 2014

HAL is a multi-disciplinary open access archive for the deposit and dissemination of scientific research documents, whether they are published or not. The documents may come from teaching and research institutions in France or abroad, or from public or private research centers.
L'archive ouverte pluridisciplinaire HAL, est destinée au dépôt et à la diffusion de documents scientifiques de niveau recherche, publiés ou non, émanant des établissements d'enseignement et de recherche français ou étrangers, des laboratoires publics ou privés. 


\title{
Three-dimensional stability of vortex arrays in a stratified and rotating fluid
}

\author{
AXEL DELONCLE†, PAUL BILLANT \\ AND JEAN-MAR C CHOMAZ \\ Hydrodynamics Laboratory (LadHyX), CNRS-Ecole Polytechnique, 91128 Palaiseau CEDEX, France
}

(Received 26 July 2010; revised 13 February 2011; accepted 7 March 2011;

first published online 12 May 2011)

This paper investigates numerically and through an asymptotic approach the threedimensional stability of steady vertical vortex arrays in a stratified and rotating fluid. Three classical vortex arrays are studied: the Kármán vortex street, the symmetric double row and the single row of co-rotating vortices. The asymptotic analysis assumes well-separated vortices and long-wavelength bending perturbations following Billant (J. Fluid Mech., vol. 660, 2010, p. 354) and Robinson \& Saffman (J. Fluid Mech., vol. 125,1982 , p. 411). Very good agreement with the numerical stability analysis is found even for finite wavelength and relatively close vortices. For a horizontal Froude number $F_{h} \leqslant 1$ and for a non-rotating fluid, it is found that the Kármán vortex street for a street spacing ratio (the distance $h$ between the rows divided by the distance $b$ between vortices in the same row) $\kappa \leqslant 0.41$ and the symmetric double row for any spacing ratio are most unstable to a three-dimensional instability of zigzag type that vertically bends the vortices. The most amplified vertical wavenumber scales like $1 /\left(b F_{h}\right)$ and the growth rate scales with the strain $\Gamma /\left(2 \pi b^{2}\right)$, where $\Gamma$ is the vortex circulation. For the Kármán vortex street, the zigzag instability is symmetric with respect to the midplane between the two rows while it is antisymmetric for the symmetric double row. For the Kármán vortex street with well-separated vortex rows $\kappa>0.41$ and the single row, the dominant instability is two-dimensional and corresponds to a pairing of adjacent vortices of the same row. The main differences between stratified and homogeneous fluids are the opposite symmetry of the dominant three-dimensional instabilities and the scaling of their most amplified wavenumber. When $F_{h}>1$, three-dimensional instabilities are damped by a viscous critical layer. In the presence of background rotation in addition to the stratification, symmetric and antisymmetric modes no longer decouple and cyclonic vortices are less bent than anticyclonic vortices. However, the dominant instability remains qualitatively the same for the three vortex arrays, i.e. quasi-symmetric or quasi-antisymmetric and three-dimensional or two-dimensional. The growth rate continues to scale with the strain but the most unstable wavenumber of three-dimensional instabilities decreases with rotation and scales like $R o /\left(b F_{h}\right)$ for small Rossby number $R o$, in agreement with quasi-geostrophic scaling laws.

Key words: instability, rotating flows, stratified flows, vortex interactions 
(a)

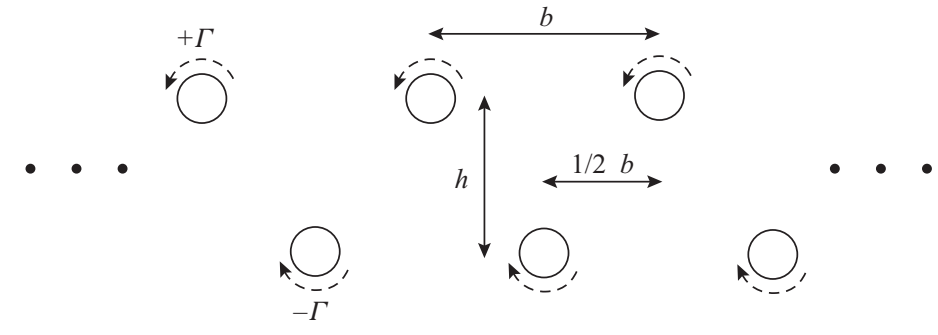

(b)
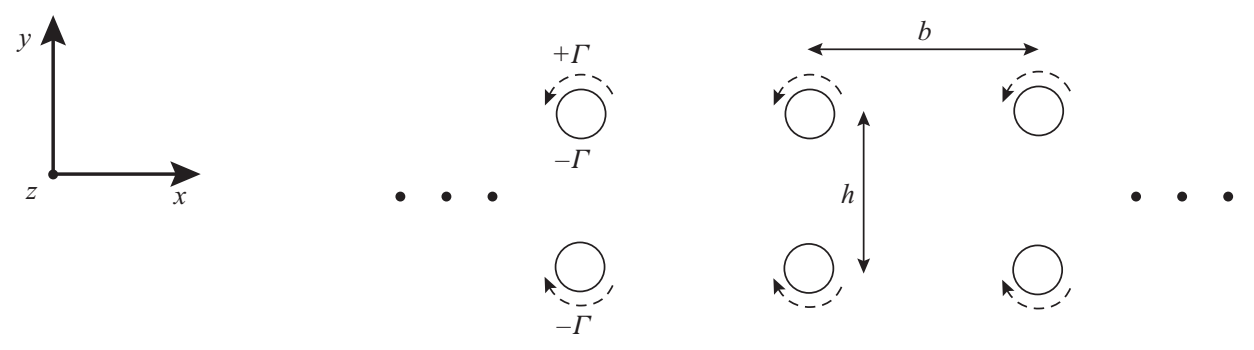

(c)
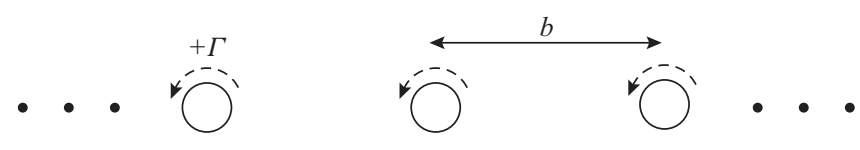

FIGURE 1. Sketch of the three vortex arrays: $(a)$ Kármán vortex street, $(b)$ symmetric double row, both characterized by the spacing ratio $\kappa=h / b,(c)$ single row. The vortices are initially two-dimensional with their axis in the vertical $z$-direction. The vortex rows are infinite along the $x$-axis.

\section{Introduction}

In this article, we investigate the three-dimensional stability in a stratified and rotating fluid of three classical vortex arrays of two-dimensional vertical vortices: (i) the Kármán vortex street, which consists of two staggered counter-rotating infinite rows of co-rotating vortices, (ii) the symmetric double row, which is similar except that the two rows are not staggered, and (iii) the single row of co-rotating vortices (figure 1).

Much attention has been devoted to their linear stability in homogeneous fluid using point vortices. This approach is valid for well-separated vortices with a reference length taken as the radius of the vortex core. The case of two-dimensional disturbances was first treated by von Kármán $(1911,1912)$ and von Kármán \& Rubach (1912), who showed that all vortex arrays were always unstable, except a single configuration of the Kármán vortex street for which the street spacing ratio $\kappa=h / b$ (the ratio of the distance $h$ between the two rows and the separation distance $b$ of the vortices in the same row, see figure 1) was equal to 0.281 . This analysis was presented in the book by Lamb (1932). 
The case of three-dimensional disturbances was later addressed by Robinson \& Saffman (1982). Their analysis was based on vortex filaments and was therefore limited to long axial wavelengths and to well-separated vortices as well. Under these hypotheses, they could make use of the Biot-Savart law to compute the induced motion of the vortices and the cutoff approximation to determine the self-induced velocity of the individual vortices. They found that the Kármán vortex street and the symmetric double row are most unstable to three-dimensional or two-dimensional disturbances depending on the spacing ratio of the streets while the single row is always most unstable to two-dimensional disturbances.

The stability of two-dimensional vortex arrays with finite cores has also been investigated in detail in a rotating fluid through numerical stability analyses (see for example Leblanc \& Cambon 1998; Potylitsin \& Peltier 1999). However, it has been less addressed in a stably stratified and rotating fluid even though these configurations can be observed in geophysical flows for instance in the wake of mountains or islands (Kármán vortex street like in Etling 1990) or as the subsequent evolution of the destabilization of a jet (symmetric double row) or a shear layer (single row). Potylitsin \& Peltier (1998) have investigated numerically the stability of an infinite vortex row in a weakly stratified and rotating fluid. They found that a weak stratification has a stabilizing effect on the elliptic instability. Experiments in a strongly stratified fluid have shown that the vortex streets created by towing a rake of vertical cylinders (Holford \& Linden 1999) or a rake of vertical flat plates (Praud, Fincham \& Sommeria 2005) are destabilized, leading to the formation of horizontal layers. The emergence of pancake vortices from a stratified horizontal shear layer perturbed by finite-amplitude fluctuations has also been recently studied numerically by Basak \& Sarkar (2006).

In contrast, the stability of a pair of columnar vertical vortices in a stratified fluid has been investigated in detail in the counter-rotating (Billant \& Chomaz 2000) and co-rotating (Otheguy, Chomaz \& Billant 2006b) cases. These studies have shown the existence of a three-dimensional instability called zigzag instability that vertically bends the vortices with little internal deformation. Billant (2010) recently derived a general theory to treat the stability of a pair of vortices for long-axial-wavelength bending perturbations and well-separated vortices in a stratified and rotating fluid. This analysis yields stability equations formally identical to those given by Crow (1970) in homogeneous fluid using the Biot-Savart law and the cutoff approximation. Only the expressions of the self-induction and mutual induction functions differ between homogeneous fluid and stratified and rotating fluid. In the present paper, we make use of this approach to study the three-dimensional stability of the Kármán vortex street, the symmetric double row and the single row for long-axial-wavelength perturbations and well-separated vortices in a stratified and rotating fluid. The asymptotic results will be contrasted to their counterpart in homogeneous fluids (Robinson \& Saffman 1982) and validated against direct numerical stability analyses. Most interestingly, we shall show that many configurations of vortex arrays in stratified and rotating fluids are unstable to the zigzag instability.

This paper is organized as follows. The governing equations are given in $\S 2$. In $\S 3$, we briefly present the asymptotic analysis of Billant (2010), before generalizing it to vortex arrays following Robinson \& Saffman (1982). The method used in the direct numerical stability analyses is described in $\S 4$. The results are presented in $\S \S 5-7: \S 5$ is first devoted to the stability of the different vortex arrays in the case of a strongly stratified and non-rotating stratified fluid. The effects of varying the stratification and the background rotation are then presented in $\S \S 6$ and 7 , respectively. Concluding remarks follow in $\S 8$. 


\section{Governing equations}

The governing equations are the incompressible Navier-Stokes equations within the Boussinesq approximation in a frame rotating at angular velocity $\Omega_{b}$ about the vertical $z$-axis:

$$
\begin{gathered}
\frac{\partial \boldsymbol{u}}{\partial t}+\boldsymbol{u} \cdot \nabla \boldsymbol{u}+2 \Omega_{b} \boldsymbol{e}_{z} \times \boldsymbol{u}=-\frac{1}{\rho_{0}} \nabla p-g \frac{\rho}{\rho_{0}} \boldsymbol{e}_{z}+v \boldsymbol{\Delta} \boldsymbol{u}, \\
\nabla \cdot \boldsymbol{u}=0, \\
\frac{\partial \rho}{\partial t}+\boldsymbol{u} \cdot \nabla \rho+\frac{\mathrm{d} \bar{\rho}}{\mathrm{d} z} w=D \Delta \rho,
\end{gathered}
$$

where $\boldsymbol{u}=(u, v, w)$ is the velocity vector in Cartesian coordinates $(x, y, z), p$ is the pressure, $\rho_{0}$ is a constant reference density, $\rho$ is the density perturbation with respect to the base density $\rho_{0}+\bar{\rho}(z), g$ is the acceleration due to gravity, $\boldsymbol{e}_{z}$ is the unit vector in the upward $z$-direction, $v$ is the kinematic viscosity, and $D$ is the molecular diffusivity of the stratifying agent. The Brunt-Väisälä frequency $N=\sqrt{-g / \rho_{0}} \mathrm{~d} \bar{\rho} / \mathrm{d} z$, measuring the density gradient, is assumed to be constant.

\section{Asymptotic analysis}

For clarity, we first present briefly the stability equations for the simple case of two vortices in a stratified and rotating fluid obtained by Billant (2010) by means of an asymptotic analysis. The generalization to several vortices is straightforward from this case.

\subsection{Interactions between a pair of vortices in a stratified and rotating fluid}

We denote by $\Gamma_{1}$ and $\Gamma_{2}$ the circulations of two vertical vortices of radius $a$ separated by a distance $b$. The Froude and Rossby numbers of each vortex are defined as follows:

$$
F_{h i}=\frac{\left|\Gamma_{i}\right|}{2 \pi a^{2} N}, \quad R o_{i}=\frac{\Gamma_{i}}{4 \pi a^{2} \Omega_{b}}, \quad \text { with } i=\{1,2\} .
$$

The asymptotic theory is based on three assumptions. First, it is assumed that the strains $\Gamma_{i} /\left(2 \pi b^{2}\right)$ are small compared to the core vorticities $\Gamma_{i} /\left(2 \pi a^{2}\right)$. This is equivalent to stating that the vortices are well-separated: $a \ll b$. The second hypothesis is that the perturbations consist of long-wavelength bending deformations of the vortices with a vertical wavenumber $k_{z}$ such that $k_{z} a F_{h i} \ll \min \left(1,\left|R o_{i}\right|\right), \max \left(1, \sqrt{F_{h i}}\right)$. These two assumptions are similar to those assumed in vortex stability analyses using vortex filaments in a homogeneous fluid (Crow 1970; Robinson \& Saffman 1982). The only difference is the small vertical wavenumber hypothesis in the homogeneous fluid which is $k_{z} a \ll 1$. The third assumption used here is $F_{h i} \ll(b / a)^{2}$, which means that the strain $\Gamma_{i} /\left(2 \pi b^{2}\right)$, exerted by one vortex on the others, is small compared to the Brunt-Väisälä frequency $N$. Since $b / a \gg 1$, this condition is fulfilled over a large range of stratifications, from strongly to weakly stratified flows. Although small viscous effects can also be taken into account within this approach, we shall consider here only the inviscid limit.

The position of each vortex centre in each horizontal plane is assumed to be perturbed by an amount $\left(\widetilde{\Delta x}_{1}(z, t), \widetilde{\Delta y_{1}}(z, t)\right)$ and $\left(\widetilde{\Delta x}_{2}(z, t), \widetilde{\Delta y_{2}}(z, t)\right)$. Writing these perturbations in the form

$$
\left(\begin{array}{c}
\widetilde{\Delta x_{i}} \\
\widetilde{\Delta y_{i}}
\end{array}\right)=\left(\begin{array}{c}
\Delta x_{i}(t) \\
\Delta y_{i}(t)
\end{array}\right) \mathrm{e}^{\mathrm{i} k_{z} z}+\text { c.c. },
$$


where c.c. denotes the complex conjugate, and using the three hypotheses mentioned above, the following evolution equations for the perturbations of the first vortex are obtained by means of an asymptotic analysis accurate at first order in $k_{z}^{2} a^{2} F_{h i}^{2}$ and $\Gamma_{i} /\left(2 \pi b^{2}\right)$ :

$$
\begin{aligned}
\frac{\mathrm{d} \Delta x_{1}}{\mathrm{~d} t}= & -\frac{\Gamma_{2}}{2 \pi b^{2}} \Delta y_{1}+\frac{\Gamma_{2}}{2 \pi b^{2}} \psi(\beta) \Delta y_{2}+\left(f-\frac{\Gamma_{1}}{2 \pi a^{2}} \omega_{\operatorname{Re}}\left(k_{z}, F_{h 1}, R o_{1}\right)\right) \Delta y_{1} \\
& +\frac{\left|\Gamma_{1}\right|}{2 \pi a^{2}} \omega_{\operatorname{Im}}\left(k_{z}, F_{h 1}, R o_{1}\right) \Delta x_{1} \\
\frac{\mathrm{d} \Delta y_{1}}{\mathrm{~d} t}= & -\frac{\Gamma_{2}}{2 \pi b^{2}} \Delta x_{1}+\frac{\Gamma_{2}}{2 \pi b^{2}} \chi(\beta) \Delta x_{2}-\left(f-\frac{\Gamma_{1}}{2 \pi a^{2}} \omega_{\operatorname{Re}}\left(k_{z}, F_{h 1}, R o_{1}\right)\right) \Delta x_{1} \\
& +\frac{\left|\Gamma_{1}\right|}{2 \pi a^{2}} \omega_{\operatorname{Im}}\left(k_{z}, F_{h 1}, R o_{1}\right) \Delta y_{1} .
\end{aligned}
$$

The complementary pair of equations for the second vortex are found by interchanging the subscripts 1 and 2 . These equations are written in the frame of reference rotating at angular velocity $f+\Omega_{b}$, where $f=\left(\Gamma_{1}+\Gamma_{2}\right) /\left(2 \pi b^{2}\right)$ is the rate at which the unperturbed vortices rotate around each other. In this frame of reference, the base flow is steady. The functions $\psi$ and $\chi$ are the mutual induction functions and $\omega=\omega_{\mathrm{Re}}+\mathrm{i} \omega_{\mathrm{Im}}$ is the self-induction function, which can be complex in a stratified and rotating fluid. They will be detailed below.

Equations (3.3) are identical to those derived by Crow (1970) or Jimenez (1975) for a pair of vortex filaments in a homogeneous fluid except that the functions $\psi$, $\chi$ and $\omega$ are different in a stratified and rotating fluid. The physical meaning of the three terms on the right-hand side of (3.3) is the following. The first term represents the strain effect of the basic flow field of one vortex on the perturbation sustained by the other vortex. The second term is the mutual induction effect, i.e. the effect of the perturbation of one vortex on the basic flow of the other vortex. This effect depends on the mutual induction functions:

$$
\chi(\beta)=-\beta^{2} \mathrm{~K}_{1}^{\prime}(\beta), \quad \psi(\beta)=\beta \mathrm{K}_{1}(\beta),
$$

where $\beta=b k_{z} F_{h 1} /\left|R o_{1}\right|=b k_{z} F_{h 2} /\left|R o_{2}\right|$ and $\mathrm{K}_{1}$ is the modified Bessel function of the second kind of first order. Although their explicit forms are different, these functions are the equivalent, in a stratified and rotating fluid, to Crow's first and second mutual induction functions (Crow 1970). Here $\chi$ and $\psi$ are equal to unity for $\beta=0$, i.e. in the two-dimensional limit $\left(k_{z}=0\right)$ and for any vertical wavenumber in the non-rotating limit. These mutual induction functions go to zero exponentially for large $\beta$.

The last term represents the effect of the rotation of the vortex pair at angular velocity $f$ and the self-induction effect, i.e. the effect of a vortex on itself. If alone, this self-induction corresponds to a rotation of the sinusoidally bent vortex at angular velocity $\Gamma_{i} /\left(2 \pi a^{2}\right) \omega_{\operatorname{Re}}$ around its unperturbed location. When $\omega_{\mathrm{Im}}<0$, this rotation is damped at rate $\sigma=|\Gamma| /\left(2 \pi a^{2}\right) \omega_{\mathrm{Im}}$. In a stratified and rotating fluid, the self-induction function (note that it is not exactly defined as in Crow 1970 or Robinson \& Saffman 1982 ) is given by

$$
\omega\left(k_{z}, F_{h i}, R o_{i}\right)=k_{z}^{2} a^{2} F_{h i}^{2}\left[\frac{\mathscr{A}\left(F_{h i}\right)}{2}+\frac{\mathscr{B}\left(F_{h i}\right)}{R o_{i}}-\frac{1}{2 R o_{i}^{2}}\left(\ln \left(\frac{k_{z} a F_{h i}}{2\left|R o_{i}\right|}\right)-\mathscr{D}\left(F_{h i}\right)+\gamma_{e}\right)\right],
$$


where $\gamma_{e}=0.5772 \ldots$ is the Euler constant and $\mathscr{A}, \mathscr{B}$ and $\mathscr{D}$ are parameters depending on the Froude number $F_{h i}$ and the non-dimensional angular velocity $\Omega$ of the individual vortices (Billant 2010). Their expressions are given in Appendix A. In this paper, we consider the Lamb-Oseen profile:

$$
\Omega(r)=\frac{1}{r^{2}}\left(1-\mathrm{e}^{-r^{2}}\right),
$$

where $r$ is the distance to the vortex axis non-dimensionalized by $a$. In this case, the self-induction is real and positive for $F_{h} \leqslant 1$ whatever $R o$ in contrast to homogeneous fluids for which it is negative. For $F_{h} \geqslant 1$, the self-induction becomes complex with a negative imaginary part $\omega_{\text {Im }}$ because the bending disturbances are damped by a viscous critical layer at the radius where the angular velocity of the vortex is equal to the Brunt-Väisälä frequency.

Billant et al. (2010) have shown that the results predicted by (3.3) and their complementary equations for the other vortex are in excellent agreement with full numerical stability analyses.

\subsection{Generalization to vortex arrays in a stratified and rotating fluid}

Robinson \& Saffman (1982) have investigated the stability of vortex arrays in a homogeneous fluid in the limit of well-separated vortices and long-wavelength bending perturbations following Crow's stability analysis of a counter-rotating vortex pair. Their stability equations have the same form as those of Crow (1970) or (3.3) except that they sum up all the strain and mutual induction effects of each vortex of the array. In order to determine the three-dimensional stability of vortex arrays in a stratified and rotating fluid, we can thus follow the analysis of Robinson \& Saffman (1982), except that the mutual induction functions and self-induction functions have to be replaced by those valid in a stratified and rotating fluid.

Since the detailed derivation of the stability equations of vortex arrays can be found in Robinson \& Saffman (1982), we recall here only briefly the main steps in the case of the Kármán vortex street. The generalization to the symmetric double row and the single row is straightforward.

\subsubsection{Kármán vortex street}

The Kármán vortex street consists of a staggered double row of two-dimensional vertical vortices. The upper row lies in the plane $y=0$, with each vortex having a positive circulation $\Gamma$ and a radius $a$ (figure 1). The lower row is in the plane $y=-h$, with each vortex having a negative circulation $-\Gamma$ and also a radius $a$. The vortices on each row are separated by a distance $b$ and the two rows are staggered by a distance $b / 2$. The whole unperturbed vortex array moves with a uniform velocity $U=\Gamma /(2 b) \tanh (\pi h / b)\left(\right.$ Lamb 1932) in the frame of reference rotating at rate $\Omega_{b}$ about the vertical axis. Since all vortices have the same absolute circulation, they are characterized by a single Froude number $F_{h}=\Gamma /\left(2 \pi a^{2} N\right)$. In contrast, the Rossby number of the vortices of the upper row is $R o=\Gamma /\left(4 \pi a^{2} \Omega_{b}\right)$ while the Rossby number of the vortices of the lower row is opposite, $-R o$.

In the frame of reference rotating at rate $\Omega_{b}$ and translating at constant velocity $U$, the position of the perturbed vortices in the horizontal plane $(x, y)$ is

$$
\begin{aligned}
& \left(x_{1, m}, y_{1, m}\right)=(m b, 0)+\left[\left(\Delta x_{1, m}, \Delta y_{1, m}\right) \mathrm{e}^{\mathrm{i} k_{z} z}+\text { c.c. }\right], \\
& \left(x_{2, m}, y_{2, m}\right)=((m+1 / 2) b,-h)+\left[\left(\Delta x_{2, m}, \Delta y_{2, m}\right) \mathrm{e}^{\mathrm{i} k_{z} z}+\text { c.c. }\right],
\end{aligned}
$$

where the subscripts $(1, m)$ and $(2, m)$ denote a vortex respectively in the upper and lower rows, $m$ being an integer. The amplitude of the three-dimensional bending 
perturbations of the vortices around their unperturbed position is $\left(\Delta x_{1, m}, \Delta y_{1, m}\right) \mathrm{e}^{\mathrm{i} k_{z} z}$ and $\left(\Delta x_{2, m}, \Delta y_{2, m}\right) \mathrm{e}^{\mathrm{i} k_{z} z}$, and $k_{z}$ is their vertical wavenumber.

By summing up the straining and the mutual induction effects due to each vortex, the following evolution equations for the perturbations of a vortex $m$ in the upper row are found:

$$
\begin{aligned}
\frac{\mathrm{d} \Delta x_{1, m}}{\mathrm{~d} t}= & -\frac{\Gamma}{2 \pi a^{2}} \omega_{\operatorname{Re}}\left(k_{z}, F_{h}, R o\right) \Delta y_{1, m}+\frac{\Gamma}{2 \pi a^{2}} \omega_{\mathrm{Im}}\left(k_{z}, F_{h}, R o\right) \Delta x_{1, m} \\
& -\frac{\Gamma}{2 \pi} \sum_{p \neq m} \frac{\Delta y_{1, m}-\psi_{p m} \Delta y_{1, p}}{b_{p m}^{2}} \\
& +\frac{\Gamma}{2 \pi} \sum_{q} \frac{\left(\tilde{b}_{q m}^{2}-h^{2}\right) \Delta y_{1, m}-\left(\tilde{b}_{q m}^{2} \psi_{q m}-h^{2} \chi_{q m}\right) \Delta y_{2, q}}{L_{q m}^{4}}, \\
\frac{\mathrm{d} \Delta y_{1, m}}{\mathrm{~d} t}= & +\frac{\Gamma}{2 \pi} \sum_{q} \frac{\Gamma \tilde{b}_{q m} h\left[2 \Delta x_{1, m}-\left(\chi_{q m}+\psi_{q m}\right) \Delta x_{2, q}\right]}{L_{q m}^{4}}, \\
& -\frac{\Gamma}{2 \pi} \sum_{p \neq m} \frac{\Delta x_{1, m}-\chi_{p m} \Delta x_{1, p}}{b_{p m}^{2}} \\
& +\frac{\Gamma}{2 \pi} \sum_{q} \frac{\left(\tilde{b}_{q m}^{2}-h^{2}\right) \Delta x_{1, m}-\left(\tilde{b}_{q m}^{2} \chi_{q m}-h^{2} \psi_{q m}\right) \Delta x_{2, q}}{L_{q m}^{4}} \\
& -\frac{\Gamma}{2 \pi} \sum_{q} \frac{\tilde{b}_{q m} h\left[2 \Delta x_{1, m}-\left(\chi_{q m}+\psi_{q m}\right) \Delta y_{2, q}\right]}{L_{q m}^{4}},
\end{aligned}
$$

where $b_{p m}=(p-m) b, \tilde{b}_{q m}=(q-m+1 / 2) b$ and $L_{q m}^{2}=h^{2}+\tilde{b}_{q m}^{2}$. The subscripts on $\psi$ and $\chi$ indicate that the function arguments are $\left|b_{p m}\right| k_{z} F_{h} /|R o|$ and $\left|L_{q m}\right| k_{z} F_{h} /|R o|$ for subscripts $p m$ and $q m$, respectively. The equations for a vortex of the lower row are found by applying the following transformations: $\Gamma \rightarrow-\Gamma, h \rightarrow-h$ and by interchanging the subscripts 1 and 2. We now consider linear perturbations of the form

$$
\begin{aligned}
& \left(\Delta x_{1, m}, \Delta y_{1, m}\right)=\left(\Delta x_{1}, \Delta y_{1}\right) \mathrm{e}^{\mathrm{i} m \phi+\sigma t}, \\
& \left(\Delta x_{2, m}, \Delta y_{2, m}\right)=\left(\Delta x_{2}, \Delta y_{2}\right) \mathrm{e}^{\mathrm{i}(m+1 / 2) \phi+\sigma t},
\end{aligned}
$$

with $\sigma$ being the growth rate and $0 \leqslant \phi \leqslant \pi$. As shown by Robinson \& Saffman (1982), $\phi / b$ can be considered as a wavenumber of the disturbance in the row direction. However, it is more convenient to use the wavelength of the disturbance $\mu b=2 \pi b / \phi$ in the row direction with $2 \leqslant \mu \leqslant \infty$. A value $\mu=2$ implies a periodicity every two vortices, $\mu=4$ every four vortices and so on, while $\mu=\infty$ means that all the vortices on a single row are displaced in the same direction.

Following Robinson \& Saffman (1982), it is also interesting to introduce symmetric and antisymmetric modes:

$$
\begin{aligned}
& \left(\Delta x_{s}, \Delta y_{s}\right)=\left(\Delta x_{1}+\Delta x_{2}, \Delta y_{1}-\Delta y_{2}\right), \\
& \left(\Delta x_{a}, \Delta y_{a}\right)=\left(\Delta x_{1}-\Delta x_{2}, \Delta y_{1}+\Delta y_{2}\right) .
\end{aligned}
$$

The physical meaning of these modes can be easily understood in the case $\mu=\infty$, i.e. when all vortices of a given row are displaced in the same direction. The symmetric 
mode then corresponds to a displacement of each row in the same $x$-direction but in the opposite $y$-direction, i.e. a vertical modulation of the row spacing $h$. Conversely, the antisymmetric mode corresponds to displacements of each row in the opposite $x$-direction but in the same $y$-direction, i.e. a vertical modulation of the interval $b / 2$ in the $x$-direction between the two rows.

Inserting (3.9) into (3.8) and the corresponding equations for the lower row, we obtain the following eigenvalue problem for the symmetric and antisymmetric modes:

$$
\boldsymbol{M} \boldsymbol{\Delta} \boldsymbol{x}=\frac{2 \pi b^{2}}{\Gamma} \sigma \boldsymbol{\Delta} \boldsymbol{x},
$$

where $\boldsymbol{\Delta} \boldsymbol{x}=\left(\Delta x_{s}, \Delta y_{s}, \Delta x_{a}, \Delta y_{a}\right)^{\top}$ and

$$
\boldsymbol{M}=\left(\begin{array}{cccc}
-B+\frac{b^{2}}{a^{2}} \bar{\omega}_{\mathrm{Im}} & -A+C-\frac{b^{2}}{a^{2}} \bar{\omega}_{\mathrm{Re}} & \frac{b^{2}}{a^{2}} \Delta \omega_{\mathrm{Im}} & -\frac{b^{2}}{a^{2}} \Delta \omega_{\mathrm{Re}} \\
-\tilde{A}-\tilde{C}+\frac{b^{2}}{a^{2}} \bar{\omega}_{\mathrm{Re}} & -\tilde{B}+\frac{b^{2}}{a^{2}} \bar{\omega}_{\mathrm{Im}} & \frac{b^{2}}{a^{2}} \Delta \omega_{\operatorname{Re}} & \frac{b^{2}}{a^{2}} \Delta \omega_{\mathrm{Im}} \\
\frac{b^{2}}{a^{2}} \Delta \omega_{\mathrm{Im}} & -\frac{b^{2}}{a^{2}} \Delta \omega_{\operatorname{Re}} & B+\frac{b^{2}}{a^{2}} \bar{\omega}_{\mathrm{Im}} & -A-C-\frac{b^{2}}{a^{2}} \bar{\omega}_{\operatorname{Re}} \\
\frac{b^{2}}{a^{2}} \Delta \omega_{\operatorname{Re}} & \frac{b^{2}}{a^{2}} \Delta \omega_{\operatorname{Im}} & -\tilde{A}+\tilde{C}+\frac{b^{2}}{a^{2}} \bar{\omega}_{\operatorname{Re}} & \tilde{B}+\frac{b^{2}}{a^{2}} \bar{\omega}_{\operatorname{Im}}
\end{array}\right) .
$$

The various parameters appearing in $\boldsymbol{M}$ are

$$
\begin{aligned}
\bar{\omega} & =\bar{\omega}_{\mathrm{Re}}+\mathrm{i} \bar{\omega}_{\operatorname{Im}}=\frac{1}{2}\left[\omega\left(k_{z}, F_{h}, R o\right)+\omega\left(k_{z}, F_{h},-R o\right)\right], \\
\Delta \omega & =\Delta \omega_{\operatorname{Re}}+\mathrm{i} \Delta \omega_{\operatorname{Im}}=\frac{1}{2}\left[\omega\left(k_{z}, F_{h}, R o\right)-\omega\left(k_{z}, F_{h},-R o\right)\right],
\end{aligned}
$$

and

$$
\begin{aligned}
& A=\frac{\pi^{2}}{3}-\frac{\pi^{2}}{\cosh ^{2} \kappa \pi}-2 \sum_{p=1}^{\infty} \frac{\psi_{p} \cos p \phi}{p^{2}} \\
& B=2 \mathrm{i} \sum_{q=0}^{\infty} \frac{\left(q+\frac{1}{2}\right) \kappa}{\left[\left(q+\frac{1}{2}\right)^{2}+\kappa^{2}\right]^{2}}\left(\chi_{q}+\psi_{q}\right) \sin \left(q+\frac{1}{2}\right) \phi \\
& C=2 \sum_{q=0}^{\infty} \frac{\left(q+\frac{1}{2}\right)^{2} \psi_{q}-\kappa^{2} \chi_{q}}{\left[\left(q+\frac{1}{2}\right)^{2}+\kappa^{2}\right]^{2}} \cos \left(q+\frac{1}{2}\right) \phi
\end{aligned}
$$

with the spacing ratio $\kappa=h / b$. The function arguments of $\psi$ and $\chi$ are $p b k_{z} F_{h} /|R o|$ and $\left[(q+1 / 2)^{2}+\kappa^{2}\right]^{1 / 2} b k_{z} F_{h} /|R o|$ for subscripts $p$ and $q$ respectively. Here $\tilde{A}, \tilde{B}$ and $\tilde{C}$ are found by interchanging the symbols $\chi$ and $\psi$ in (3.15).

Contrary to homogeneous fluids (Robinson \& Saffman 1982), the symmetric and antisymmetric modes do not always decouple in (3.12) because the self-induction functions of the vortices of the upper and lower rows are not equal for finite $R o$ : $\Delta \omega \neq 0$. A decoupling is recovered only in the limits $R o \rightarrow \infty$ and $R o \rightarrow 0$ for which $\omega\left(k_{z}, F_{h}, R o\right)=\omega\left(k_{z}, F_{h},-R o\right)($ see $(3.5))$.

\subsubsection{Symmetric double row}

The symmetric double row translates at a velocity $U=\Gamma /(2 b) \operatorname{coth}(\pi h / b)$. In the frame rotating at rate $\Omega_{b}$ and translating at a constant velocity $U$, the equations for the perturbations are the same as $(3.8)$, except that $\tilde{b}_{q m}=(q-m) b$. Considering 
perturbations of the form

$$
\begin{aligned}
& \left(\Delta x_{1, m}, \Delta y_{1, m}\right)=\left(\Delta x_{1}, \Delta y_{1}\right) \mathrm{e}^{\mathrm{i} m \phi+\sigma t}, \\
& \left(\Delta x_{2, m}, \Delta y_{2, m}\right)=\left(\Delta x_{2}, \Delta y_{2}\right) \mathrm{e}^{\mathrm{i} m \phi+\sigma t},
\end{aligned}
$$

leads to exactly the same eigenvalue problem as (3.12), except that the coefficients $A, B$ and $C$ in the matrix $\boldsymbol{M}$ are slightly different:

$$
\begin{aligned}
& A=\frac{\pi^{2}}{3}+\frac{\pi^{2}}{\sinh ^{2} \kappa \pi}-2 \sum_{p=1}^{\infty} \frac{\psi_{p} \cos p \phi}{p^{2}}, \\
& B=2 \mathrm{i} \sum_{q=1}^{\infty} \frac{q \kappa}{\left(q^{2}+\kappa^{2}\right)^{2}}\left(\chi_{q}+\psi_{q}\right) \sin q \phi, \\
& C=-\frac{\chi_{\kappa}}{\kappa^{2}}+2 \sum_{q=1}^{\infty} \frac{q^{2} \psi_{q}-\kappa^{2} \chi_{q}}{\left(q^{2}+\kappa^{2}\right)^{2}} \cos q \phi,
\end{aligned}
$$

where the function arguments of $\psi$ and $\chi$ are $p b k_{z} F_{h} /|R o|,\left(q^{2}+\kappa^{2}\right)^{1 / 2} b k_{z} F_{h} /|R o|$ and $\kappa b k_{z} F_{h} /|R o|$ for subscripts $p, q$ and $\kappa$, respectively. Here $\tilde{A}, \tilde{B}$ and $\tilde{C}$ are also found by interchanging the symbols $\chi$ and $\psi$ in (3.18).

\subsubsection{Single row}

Finally, the case of the single row can be directly obtained by neglecting all terms related to the second row in (3.12) and $\boldsymbol{M}$ and by taking the limit $\kappa \rightarrow \infty$ for the coefficients in (3.15):

$$
\left(\begin{array}{cc}
\frac{b^{2}}{a^{2}} \omega_{\operatorname{Im}} & -A-\frac{b^{2}}{a^{2}} \omega_{\operatorname{Re}} \\
-\tilde{A}+\frac{b^{2}}{a^{2}} \omega_{\operatorname{Re}} & \frac{b^{2}}{a^{2}} \omega_{\operatorname{Im}}
\end{array}\right)\left(\begin{array}{c}
\Delta x_{1} \\
\Delta y_{1}
\end{array}\right)=\frac{2 \pi b^{2}}{\Gamma} \sigma\left(\begin{array}{c}
\Delta x_{1} \\
\Delta y_{1}
\end{array}\right),
$$

with

$$
A=\frac{\pi^{2}}{3}-2 \sum_{p=1}^{\infty} \frac{\psi_{p} \cos p \phi}{p^{2}}
$$

where the function argument of $\psi_{p}$ is $p b k_{z} F_{h} /|R o|$. Here $\tilde{A}$ has the same expression as $A$ but with $\psi$ replaced by $\chi$. In this case, the growth rate can be obtained directly:

$$
\frac{2 \pi b^{2}}{\Gamma} \sigma= \pm \sqrt{\left(A+\frac{b^{2}}{a^{2}} \omega_{\operatorname{Re}}\right)\left(\tilde{A}-\frac{b^{2}}{a^{2}} \omega_{\operatorname{Re}}\right)}+\frac{b^{2}}{a^{2}} \omega_{\operatorname{Im}} .
$$

\section{Direct numerical stability analysis}

In order to check the theoretical results, the dominant instability of the three vortex array configurations has been determined by means of the following numerical method.

\subsection{Computation of the basic states}

The basic flows corresponding to the different vortex arrays have been first computed thanks to two-dimensional nonlinear numerical simulations initialized by vortices placed in the required arrangement and each having a Lamb-Oseen profile. For 


\begin{tabular}{cccccccccccc}
\hline Label & Configuration & $a$ & $\Gamma$ & $b$ & $\kappa$ & $R e$ & $L_{x}$ & $L_{y}$ & $N_{x}$ & $N_{y}$ & $\delta_{t}$ \\
K0.2 & Kármán vortex street & 1 & $2 \pi$ & 15 & 0.2 & 50000 & 30 & 38 & 512 & 640 & 0.01 \\
K0.5 & Kármán vortex street & 1 & $2 \pi$ & 15 & 0.5 & 50000 & 30 & 38 & 512 & 640 & 0.01 \\
D0.5 & Symmetric double row & 1 & $2 \pi$ & 15 & 0.5 & 50000 & 30 & 38 & 512 & 640 & 0.01 \\
S & Single row & 1 & $2 \pi$ & 15 & - & 50000 & 30 & 60 & 512 & 1024 & 0.01 \\
K0.2b10 & Kármán vortex street & 1 & $2 \pi$ & 10 & 0.2 & 50000 & 20 & 38 & 384 & 640 & 0.01 \\
K0.2b7.5 & Kármán vortex street & 1 & $2 \pi$ & 7.5 & 0.2 & 50000 & 15 & 38 & 256 & 640 & 0.01
\end{tabular}

TABLE 1. Overview of the physical and numerical parameters of the basic states.

example, the initial distribution of the vertical vorticity for the Kármán vortex street is $\zeta(x, y, t=0)=\sum_{m}\left(\zeta_{1, m}+\zeta_{2, m}\right)$, where $\zeta_{1, m}$ and $\zeta_{2, m}$, the dimensional basic vertical vorticities of the vortices of the upper and lower row, respectively, are given by

$$
\begin{aligned}
& \zeta_{1, m}=\frac{\Gamma}{\pi a^{2}} \exp \left[-\frac{(x-m b)^{2}+y^{2}}{a^{2}}\right], \\
& \zeta_{2, m}=-\frac{\Gamma}{\pi a^{2}} \exp \left[-\frac{\left(x-\left(m+\frac{1}{2}\right) b\right)^{2}+(y+h)^{2}}{a^{2}}\right] .
\end{aligned}
$$

Only the inviscid limit of the theory will be considered so that the Reynolds number in the computations has been set to a large value $R e=\Gamma /(2 \pi v)=50000$. As time evolves, each vortex rapidly adapts to the strain exerted by each other so that a quasi-steady state is quickly reached. Since the Reynolds number is large, $a$ and $\Gamma$ remain almost constant. The Froude number $F_{h}$ and the Rossby number $R o$ are therefore based on the initial circulation $\Gamma$ and vortex radius $a$.

The numerical simulations are performed with a pseudo-spectral method with periodic boundary conditions. Time advancement is carried out using the classical fourth-order Runge-Kutta scheme for the nonlinear term and exact integration for the viscous term (see Vincent \& Meneguzzi 1991 for details). Most of the aliasing is removed by applying a square truncation keeping $9 / 10$ of the Fourier modes along each direction. Since the base flow is periodic, the computational domain size in the row direction $L_{x}$ is taken as $2 b$, in order to compute two periods of the base flow. As explained in the next subsection, such a choice enables the computation of the stability properties for only two values of $\mu: \infty$ and 2 . In the other direction, $L_{y}$ is taken large enough to minimize the effect of the periodic boundary condition. The number of collocation points $N_{x}$ and $N_{y}$ is chosen in order to have the same mesh resolution in both directions. The time step is $\delta_{t}=0.01$ in all simulations. The numerical parameters of all the basic states are summarized in table 1.

\subsection{Three-dimensional stability problem}

The two-dimensional basic states with velocity $\boldsymbol{U}$ and pressure $P$ are subjected to infinitesimal three-dimensional perturbations such that the total flow is of the form

$$
\left(\begin{array}{c}
\boldsymbol{u} \\
p \\
\rho
\end{array}\right)(x, y, z, t)=\left(\begin{array}{c}
\boldsymbol{U} \\
P \\
0
\end{array}\right)(x, y)+\left(\begin{array}{c}
\tilde{\boldsymbol{u}} \\
\tilde{p} \\
\tilde{\rho}
\end{array}\right)(x, y, t) \mathrm{e}^{\mathrm{i} k_{z} z+\mathrm{i} \alpha x}+\text { c.c. },
$$

where quantities with a tilde denote the perturbations and have a period $b$ in the $x$-direction, $k_{z}$ is the vertical wavenumber and $\alpha=2 \pi /(\mu b)$ measures the periodicity of the perturbation along the row-wise direction. The quantity $\mu$ has the same meaning 
as in $\S 3.2$. The flow decomposition (4.2) is inserted in (2.1) and the equations are linearized around the base state.

The linearized equations are integrated numerically for each $k_{z}$ and $\mu$ value using a linearized pseudo-spectral code similar to that described in $\S 4.1$. Here, we have restricted the analysis to the values $\mu=\infty$ (fundamental mode) and $\mu=2$ (subharmonic mode), for which the theory predicts the dominant modes to exist. In practice, the overall most unstable mode for these two values of $\mu$ can be obtained from a single simulation with the length of the computational domain set to $L_{x}=2 b$, i.e. two periods of the base state. The size of the computational domain in the $y$-direction $L_{y}$ and the number of collocation points $N_{x}, N_{y}$ are identical to those used to compute the corresponding basic state $\boldsymbol{U}$. The Schmidt number $S c=v / D$ is set to unity, $S c=1$. The perturbation velocity $\tilde{\boldsymbol{u}}(x, y, t=0)$ is initialized with a divergence-free white noise. After integrating the linearized equations for a sufficiently long time, typically $T=2400 \pi a^{2} / \Gamma$, the eigenmode with the largest growth rate dominates over the perturbation and we can retrieve its structure and growth rate. Since the asymptotic analysis is valid when the vortices are well-separated, we have first investigated in the computations a small ratio of the core radius of the vortices over their separation distance $a / b=0.067$. This corresponds to the basic states labelled $\mathrm{K} 0.2, \mathrm{~K} 0.5, \mathrm{D} 0.5$ and $\mathrm{S}$ in table 1 . The effective ratio $a / d$, where $d$ is the minimum distance between vortices, is however larger and reaches a value as large as $a / d=0.133$ for the symmetric double row for $\kappa=0.5$. Larger values of the ratio $a / b$ will be considered in Appendix B (basic states $\mathrm{K} 0.2 \mathrm{~b} 10$ and $\mathrm{K} 0.2 \mathrm{~b} 7.5$ in table 1).

\section{Strongly stratified and non-rotating fluid}

For clarity, we start by presenting the case of a strongly stratified and non-rotating fluid for each of the three vortex arrays. This particular case is representative and will serve as a reference to describe the effects of the Froude and Rossby numbers presented in $\$ \S 6$ and 7.

\subsection{Kármán vortex street}

In the case of a stratified and non-rotating fluid $(R o \rightarrow \infty)$, additional simplifications can be made in the asymptotic eigenvalue problem (3.12). The arguments of the mutual induction functions are equal to 0 , implying that $\chi$ and $\psi$ are always equal to unity. This also implies that $A=\tilde{A}, B=\tilde{B}$ and $C=\tilde{C}$. Moreover, we see from (3.5) that the self-induction functions $\omega\left(k_{z}, F_{h}, \pm R o\right)$ tend to a single function $\omega_{\infty}\left(k_{z}, F_{h}\right)$ when $R o \rightarrow \infty$ :

$$
\omega_{\infty}\left(k_{z}, F_{h}\right)=\lim _{R o \rightarrow \infty} \omega\left(k_{z}, F_{h}, \pm R o\right)=\frac{\mathscr{A}\left(F_{h}\right)}{2} k_{z}^{2} a^{2} F_{h}^{2}
$$

The symmetric and antisymmetric modes then decouple since $\Delta \omega_{\mathrm{Re}}=\Delta \omega_{\mathrm{Im}}=0$ in (3.12). Their growth rate is given by

$$
\begin{aligned}
& \frac{2 \pi b^{2}}{\Gamma} \sigma_{s}=-B \pm \sqrt{A^{2}-\left(C-\frac{b^{2}}{a^{2}} \omega_{\infty \mathrm{Re}}\right)^{2}}+\frac{b^{2}}{a^{2}} \omega_{\infty \mathrm{Im}} \\
& \frac{2 \pi b^{2}}{\Gamma} \sigma_{a}=+B \pm \sqrt{A^{2}-\left(C+\frac{b^{2}}{a^{2}} \omega_{\infty \mathrm{Re}}\right)^{2}}+\frac{b^{2}}{a^{2}} \omega_{\infty \mathrm{Im}}
\end{aligned}
$$


(a)

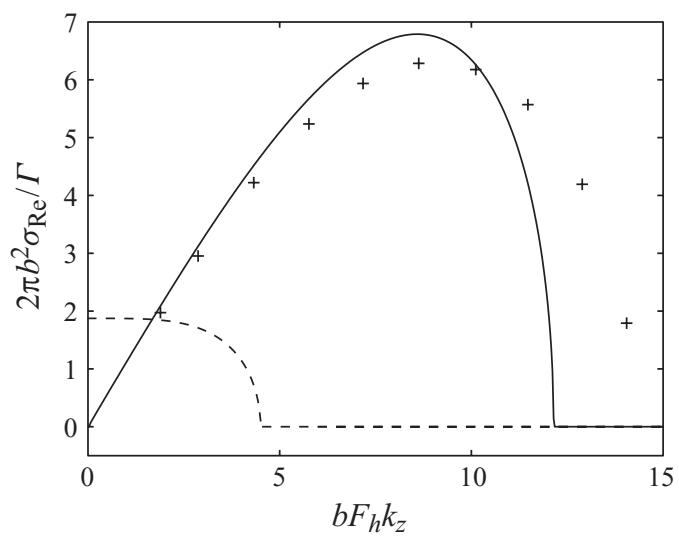

(b)

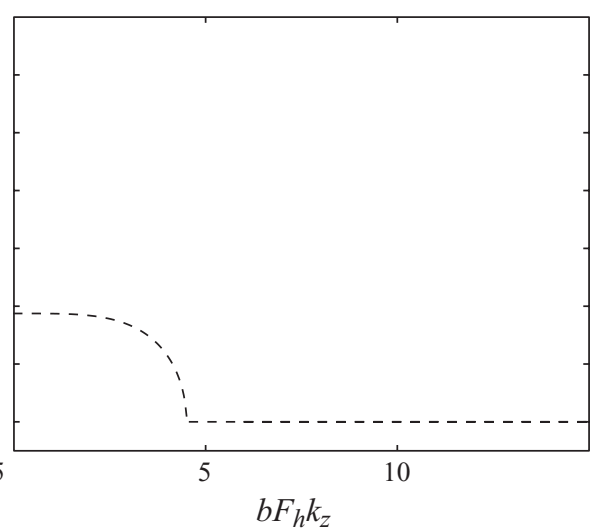

FiguRE 2. Non-dimensional growth rate $2 \pi b^{2} \sigma_{\mathrm{Re}} / \Gamma$ as a function of the rescaled vertical wavenumber $b F_{h} k_{z}$ for the Kármán vortex street with close rows $\kappa=0.2$ in a strongly stratified $\left(F_{h}=0.1\right)$ and non-rotating fluid: $(a)$ symmetric mode and $(b)$ antisymmetric mode. The growth rate for $\mu=\infty$ and $\mu=2$ is plotted by solid and dashed lines, respectively. The symbols $(+)$ correspond to the growth rate of the dominant mode obtained from the numerical stability analyses (symmetric, $\mu=\infty$ ).

where $A, B$ and $C$ are given by (3.15) with $\chi=\psi=1$. Because $B$ is purely imaginary and $\omega_{\infty}$ Im $\leqslant 0,(5.2)$ shows that the vortex array can be unstable only if $A^{2}>(C \pm$ $\left.\left(b^{2} / a^{2}\right) \omega_{\infty \mathrm{Re}}\right)^{2}$. In the two-dimensional limit $k_{z}=0$, we have $\omega_{\infty}=0$ so that symmetric and antisymmetric modes have the same growth rate. Similarly, symmetric and antisymmetric modes have the same growth rate whatever $k_{z}$ when $\mu=2$ because $C=0$.

Before presenting our results, it is interesting to recall briefly the results of Robinson \& Saffman (1982) for the Kármán vortex street in homogeneous fluids. They found that for $\kappa$ less than about 0.3 (the precise value depends on the vortex radius $a$ compared to $b$ ), the dominant instability is three-dimensional with $\mu=\infty$ and with an antisymmetric configuration (we exclude the short-wavelength instabilities also found by Robinson \& Saffman 1982 but for which the filament approach is not valid). When $\kappa$ is greater than about 0.3 , the most unstable mode is two-dimensional with $\mu=2$ and with either the symmetric and antisymmetric configurations since they have the same growth rate in this case (see (5.2)). This two-dimensional instability is a pairing instability where adjacent vortices of the same row tend to merge. Now looking at the case of a strongly stratified $\left(F_{h}=0.1\right)$ and non-rotating fluid, figure 2 shows the real part of the non-dimensional growth rate $2 \pi b^{2} \sigma_{\mathrm{Re}} / \Gamma$ of the symmetric and antisymmetric modes as a function of the rescaled vertical wavenumber $b F_{h} k_{z}$ for the street spacing ratio $\kappa=0.2$. The theoretical results are shown both for the $\mu=\infty$ (solid line) and the $\mu=2$ (dashed line) modes whereas the numerical method provides solely the growth rate of the dominant mode (symbols), i.e. in the present case the $\mu=\infty$ symmetric instability. For $\mu=\infty$, we see that the symmetric mode is most unstable for a finite value of $k_{z}$ while the antisymmetric mode is stable for all vertical wavenumbers. Strikingly, this is the opposite to the case of a homogeneous fluid where the symmetric mode is stable while the antisymmetric mode is threedimensionally unstable. As seen in figure 2(a), the growth rate of the most unstable mode is in perfect agreement for small values of $k_{z}$ with the growth rate obtained from the numerical stability analysis (symbols). The theory predicts very well the 
(a)

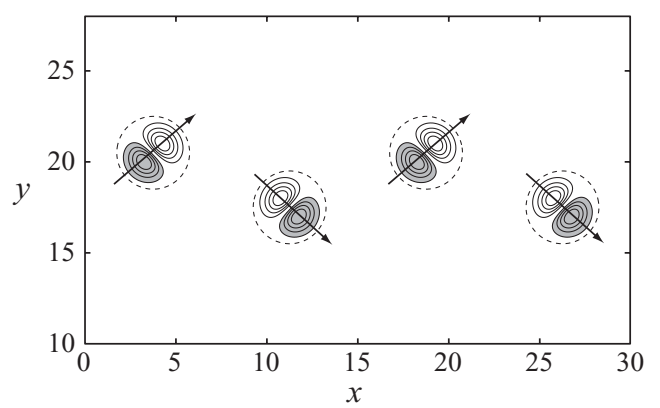

(b)

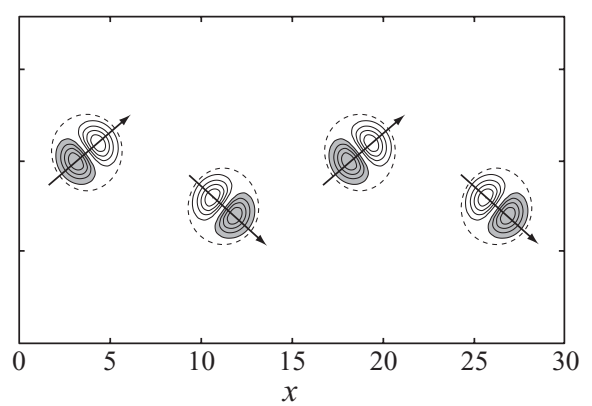

FIGURE 3. Contours of the vertical vorticity $\widetilde{\zeta}$ of the most amplified perturbation in a strongly stratified $\left(F_{h}=0.1\right)$ and non-rotating fluid of the Kármán vortex street with close rows $\kappa=0.2$. Plot $(a)$ corresponds to the asymptotic theory and $(b)$ corresponds to the numerical results. The arrows indicate the direction of displacement of each vortex that alternates along the vertical $z$-direction. The dominant instability is a three-dimensional symmetric zigzag instability with $\mu=\infty$. Only a domain of size $30 \times 18$ is shown while the original computational domain is $30 \times 38$. Shaded areas are regions of negative values. The dotted line indicates the boundary $r=2$ of the vortices of the basic state.

most unstable vertical wavenumber $k_{z \max }$ and the maximum growth rate but small departures at large wavenumbers are observed because the asymptotic theory is restricted to long-wavelength disturbances. Figure 3(a) shows the vertical vorticity field $\widetilde{\zeta}$ of the most amplified perturbation predicted theoretically. It is given at leading order by

$$
\widetilde{\zeta}=-\sum_{m}\left[\Delta x_{1, m} \frac{\partial \zeta_{1, m}}{\partial x}+\Delta y_{1, m} \frac{\partial \zeta_{1, m}}{\partial y}+\Delta x_{2, m} \frac{\partial \zeta_{2, m}}{\partial x}+\Delta y_{2, m} \frac{\partial \zeta_{2, m}}{\partial y}\right],
$$

where $\zeta_{1, m}$ and $\zeta_{2, m}$ are the dimensional basic vertical vorticities of the vortices of the upper and lower rows, respectively (see (4.1)). It is in full agreement with the dominant eigenmode determined numerically (figure $3 b$ ). We see that the perturbations are localized in the vortex cores and consist for each vortex of two regions of opposite vorticity. Such perturbations correspond to translations of the vortices in the direction indicated by the arrows. The displacements are symmetric with respect to the midplane between the two rows and all the vortices of a given row are displaced in the same direction since $\mu=\infty$. A three-dimensional sketch of the perturbed vortices is presented in figure 4. Because such a three-dimensional bending instability in strongly stratified fluids has been called a zigzag instability for vortex pairs (Billant \& Chomaz 2000; Otheguy et al. 2006b), we also use this term for the present threedimensional symmetric instability of the Kármán vortex street in stratified fluids. In homogeneous fluids, the instability is antisymmetric so that it is the interval in the $x$-direction between the two rows which is vertically modulated, as shown by the sketch in figure 5(a). For a counter-rotating vortex pair, a similar inversion of the symmetry is observed: the unstable mode is symmetric in homogeneous fluids (Crow instability), while in stratified fluids, it is the antisymmetric mode which is unstable (zigzag instability) (Billant et al. 2010). This inversion is because the self-induction is positive in stratified and rotating fluids while it is negative in homogeneous fluids. Indeed, since $C=-A>0$ for $\mu=\infty$ and $R o=\infty$, we see directly from (5.2) that the symmetric mode is unstable and the antisymmetric mode is stable only because $\omega_{\infty \mathrm{Re}}>0$. 


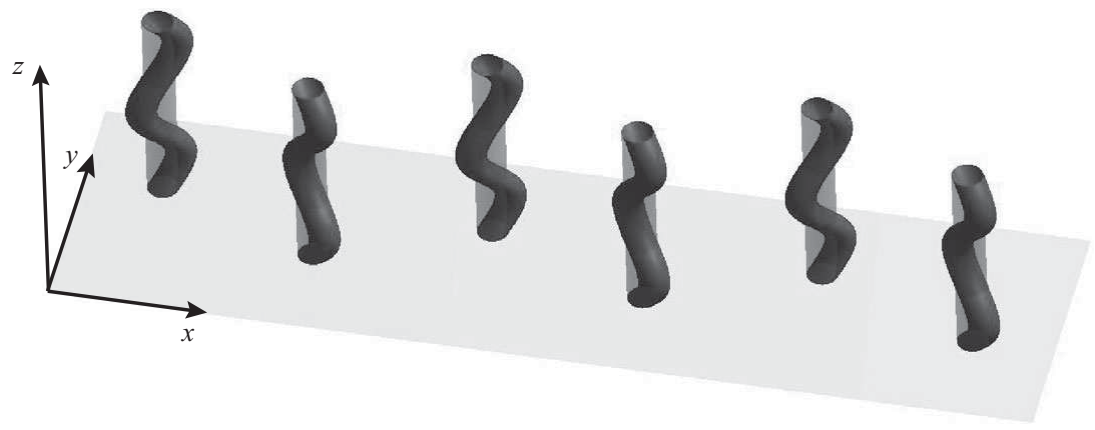

FiguRE 4. Three-dimensional sketch of the theoretical dominant instability of the Kármán vortex street with close rows $\kappa=0.2$ in a strongly stratified $\left(F_{h}=0.1\right)$ and non-rotating fluid. The unperturbed vortices are shown by light grey. The perturbed vortices, shown in dark grey, have been obtained by adding to the basic state the most unstable theoretical eigenmode with a small but finite amplitude. The dominant mode is a $\mu=\infty$ symmetric zigzag instability that vertically bends the vortices. Only one and a half vertical wavelengths are plotted.

(a)

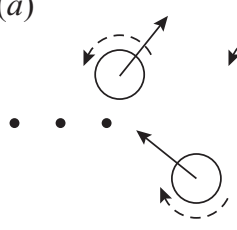

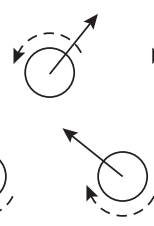

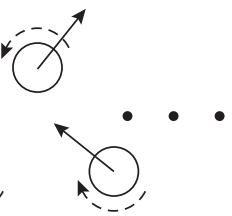

(b)

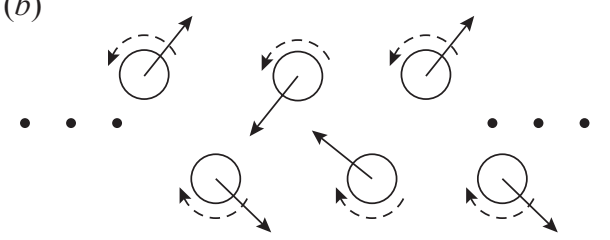

FIGURE 5. Sketch of the displacements in horizontal cross-sections induced by the two different dominant modes of the Kármán vortex street in homogeneous fluids. (a) Antisymmetric three-dimensional instability $(\mu=\infty)$ existing in homogeneous fluids (Robinson \& Saffman $1982)$ and $(b)$ symmetric pairing mode $(\mu=2)$ existing in homogeneous and strongly stratified fluids. In the case of three-dimensional instabilities, the direction of the displacements alternates along the vertical $z$-direction.

As seen in figure 2, the growth rate for $\mu=2$ (dashed line) is also positive but the maximum growth rate is obtained for two-dimensional perturbations $\left(k_{z}=0\right)$ and the symmetric and antisymmetric modes have the same growth rate. As shown in figure $5(b)$, this instability is a pairing instability which tends to move closer or away from adjacent vortices of a given row (von Kármán 1912; Robinson \& Saffman 1982). Because this instability is two-dimensional, the stratification has no effect on the dominant mode. However, note that the growth rate of three-dimensional perturbations decreases with $b F_{h} k_{z}$ in strongly stratified fluids instead of $b k_{z}$ in homogeneous fluids. As $F_{h} \rightarrow 0$, the band of unstable vertical wavenumbers $k_{z}$ thus widens as for the instability of a horizontal shear layer in stratified fluids (Deloncle, Chomaz \& Billant 2007). It is also worth noting that the maximum growth rate is significantly lower for $\mu=2$ than for $\mu=\infty$. When $\mu$ is varied between 2 and $\infty$, the most amplified vertical wavenumber and the maximum growth rate increase monotonically. The overall dominant instability for the spacing ratio $\kappa=0.2$ is therefore the symmetric three-dimensional instability for $\mu=\infty$.

Figure 6 summarizes the characteristics of the dominant instabilities when $\kappa$ is varied. The theoretically predicted maximum growth rate $2 \pi b^{2} \sigma_{\mathrm{Re}}^{M} / \Gamma$, the most amplified wavenumber $b F_{h} k_{z}^{M}$ and row-wise periodicity $\mu^{M}$ are plotted as a function of $\kappa$ for the symmetric and antisymmetric modes. We see that the $\mu=\infty$ antisymmetric three-dimensional instability is the dominant instability for $\kappa \leqslant 0.41$ (note that this 
(a)

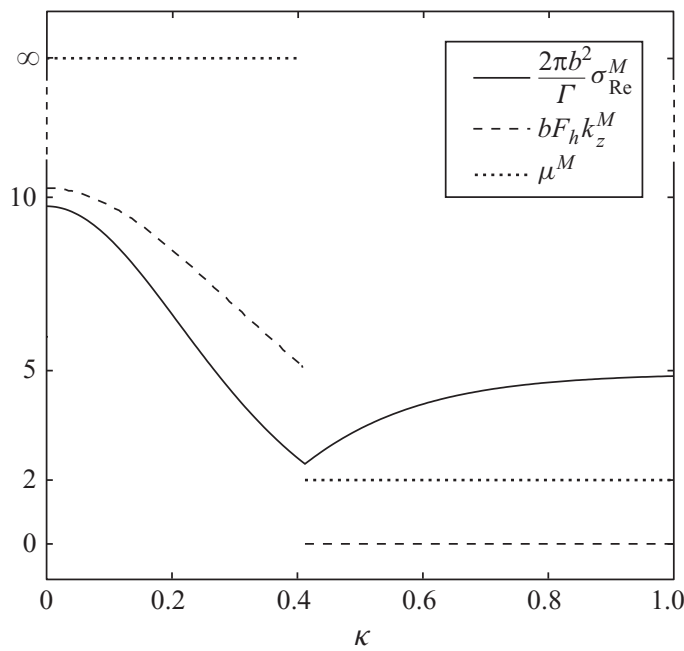

(b)

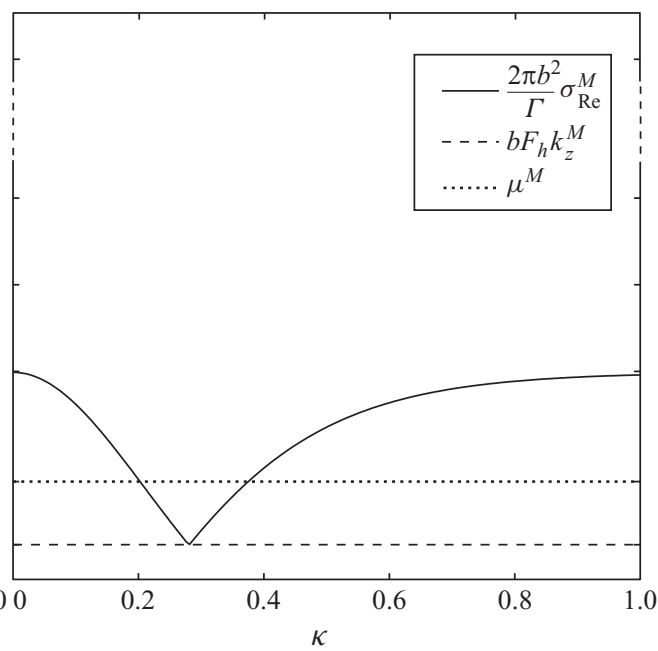

FIGURE 6. Plots of the maximum growth rate $2 \pi b^{2} \sigma_{\mathrm{Re}}^{M} / \Gamma$, the corresponding wavenumbers $b F_{h} k_{z}^{M}$ and row-wise periodicity $\mu^{M}$ versus the street spacing ratio $\kappa$ for the Kármán vortex street in a strongly stratified $\left(F_{h}=0.1\right)$ and non-rotating fluid: $(a)$ symmetric mode and (b) antisymmetric mode.

value is independent of the ratio $a / b$ in a stratified and non-rotating fluid). When the two vortex rows are more distant $\kappa>0.41$, the dominant overall instability becomes the $\mu=2$ two-dimensional instability (pairing instability) with both symmetric and antisymmetric configurations. Interestingly, the spacing ratio of vortex streets behind two-dimensional bluff bodies lies usually within the range $\kappa<0.41$, so that they might be unstable to the zigzag instability in stratified fluids.

\subsection{Symmetric double row}

In the case of the symmetric double row in a stratified and non-rotating fluid, simplifications similar to the Kármán vortex street case are made. Symmetric and antisymmetric modes decouple, leading to the same dispersion relations (5.2) with $\omega$ equal to (5.1) and $A, B$ and $C$ given by (3.18) by taking again $\psi$ and $\chi$ equal to 1 .

In homogeneous fluid, Robinson \& Saffman (1982) have found that the most unstable instability for this vortex array is always the symmetric mode for $\mu=2$ and is three-dimensional regardless of the value of the street spacing ratio $\kappa$.

As shown in figure 7 , the most unstable mode of the symmetric double row for $\kappa=0.5$ in a strongly stratified $\left(F_{h}=0.1\right)$ and non-rotating fluid is also predicted to be three-dimensional with $\mu=2$ (dashed line in figure $7 b$ ) like in homogeneous fluid but with the opposite symmetry, i.e. antisymmetric. The agreement between numerical (symbols) and theoretical (solid lines) results is again excellent for small wavenumbers and good for large wavenumbers. The antisymmetric mode for $\mu=\infty$ (solid line in figure $7 b$ ) is also three-dimensionally unstable but its maximum growth rate is much lower than for $\mu=2$. The symmetric mode is unstable for $\mu=2$ but not for $\mu=\infty$ (figure $7 a$ ).

The vertical vorticity field of the theoretical most amplified perturbation is displayed in figure $8(a)$. It is also given by $(5.3)$ but with $(m+1 / 2)$ replaced by $m$ in $\zeta_{2, m}$. The agreement with the most unstable eigenmode determined numerically is excellent (figure $8 b$ ). We see that two adjacent vortices of a given row tend to move closer 
(a)

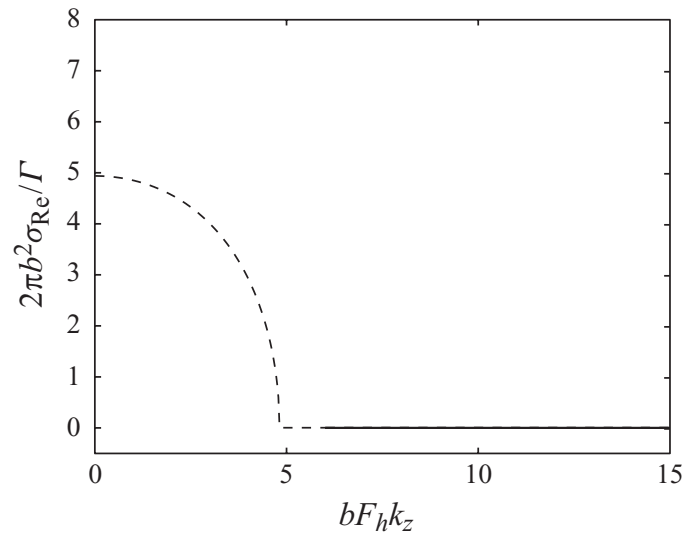

(b)

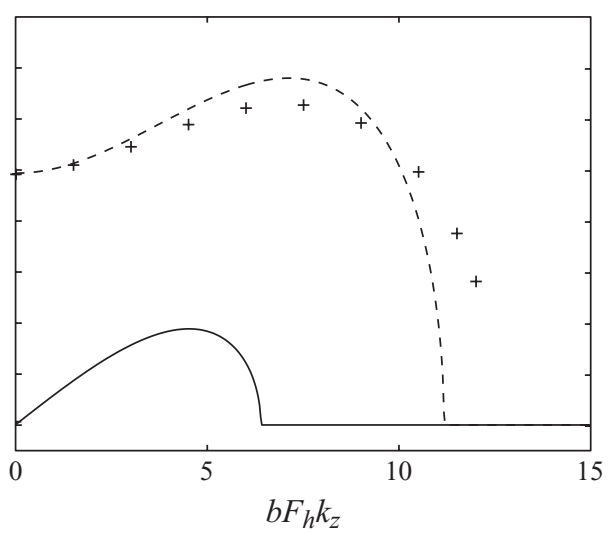

FiguRE 7. Similar to figure 2 but for the double symmetric row with $\kappa=0.5$. (a) Symmetric mode and $(b)$ antisymmetric mode. We recall that two row-wise periodicities are displayed: $\mu=\infty$ (solid lines) and $\mu=2$ (dashed lines). The symbols $(+)$ correspond to the numerical results found for the dominant mode (antisymmetric, $\mu=2$ ).

(a)

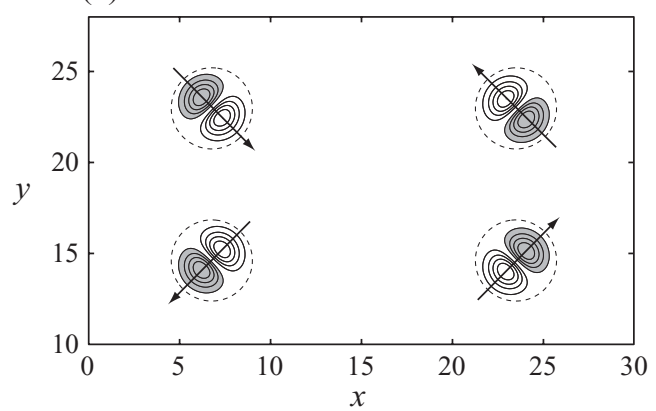

(b)

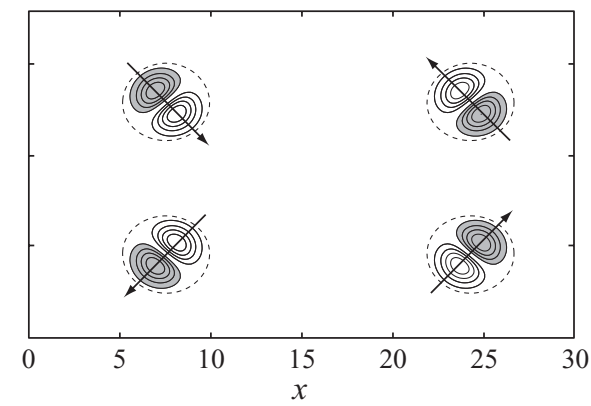

FIGURE 8. Similar to figure 3 but for the symmetric double row for $\kappa=0.5$. (a) Symmetric mode and $(b)$ antisymmetric mode.

to or farther from each other, like in the pairing instability since $\mu=2$. Similarly, two facing vortices of the two rows are displaced antisymmetrically and tend to be rotated like in the zigzag instability of a single counter-rotating vortex pair (Billant \& Chomaz 2000).

Figure 9 shows the maximum growth rate of the dominant instability over all possible values of $\mu$ as a function of $\kappa$ for the symmetric and antisymmetric configurations. The $\mu=2$ antisymmetric instability is the most unstable for all values of $\kappa$. In the limit where the rows are very close, i.e. when $\kappa \rightarrow 0$, the growth rate scaled by $\Gamma /\left(2 \pi b^{2}\right)$ diverges since it is proportional to the maximum strain: $\sigma_{\mathrm{Re}}^{M} \propto \Gamma /\left(2 \pi h^{2}\right)$ so that $\sigma_{\mathrm{Re}}^{M} 2 \pi b^{2} / \Gamma \propto 1 / \kappa^{2}$. When $\kappa$ increases, i.e. when the distance between the two rows increases, the most amplified vertical wavenumber $k_{z}^{M}$ tends to 0 . This is because the unstable interaction between the facing rows of vortices decreases so that the $\mu=2$ three-dimensional zigzag instability tends towards a two-dimensional pairing instability of adjacent vortices of a given row. 
(a)

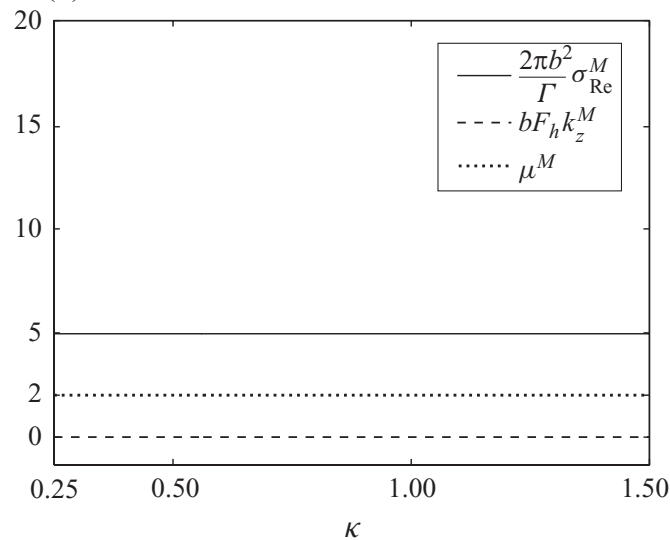

(b)

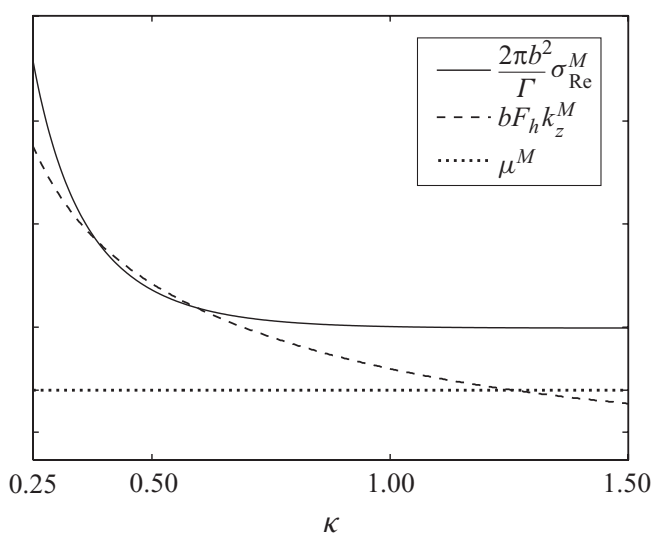

FIGURE 9. Similar to figure 6 but for the symmetric double row. (a) Symmetric mode and $(b)$ antisymmetric mode.

\subsection{Single row}

The limit $\kappa \rightarrow \infty$ considered above for the Kármán vortex street or the symmetric double row is in fact identical to the case of a single row since the two rows are infinitely far away from each other and do not interact. As explained above, the dominant instability is then a two-dimensional instability with $\mu=2$, i.e. a pairing instability of adjacent vortices of the row.

\section{Stratified and non-rotating fluid: effect of the Froude number}

We now investigate the effect of the Froude number, still for a non-rotating fluid. The growth rate formulae (5.2) for the Kármán vortex street and their equivalent for the symmetric double row and the single row remain valid.

\subsection{Kármán vortex street}

Figure 10 shows the theoretical growth rate for the Kármán vortex street of the symmetric and antisymmetric modes for various Froude numbers from strong stratification $\left(F_{h}=0.1\right)$ to moderate stratification $\left(F_{h}=1.2\right)$. For each Froude number, the agreement between the growth rate of the dominant mode $(\mu=\infty$ symmetric) obtained numerically (symbols) and theoretically (solid lines) is excellent at small rescaled wavenumbers and satisfactory at large wavenumbers. The numerical stability analysis predicts a slightly larger band of unstable wavenumber than the theory. We see that the growth rate curves (solid lines) of the three-dimensional zigzag instability for $F_{h}=0.1$ and $F_{h}=1$ are almost similar except for a small decrease of the most amplified wavenumber. Equations (5.1) and (5.2) show that the growth rate $\sigma_{\mathrm{Re}}$ is a function of $F_{h}$ and $k_{z}$ only through the product $b F_{h} k_{z} \sqrt{\mathscr{A}\left(F_{h}\right)}$, when $\mathscr{A}$ is purely real, i.e. when $F_{h}<1$ (see Appendix A). Consequently, the maximum theoretical growth rate is independent of $F_{h}$ when $F_{h}<1$. For this reason, for all $F_{h}<1$, the threshold $\kappa=0.41$, below which the dominant instability is three-dimensional, is the same as for $F_{h}=0.1$. The stratification has thus little effect on the zigzag instability as long as $F_{h} \leqslant 1$. However, when the Froude number is further increased to $F_{h}=1.2$, an abrupt drop of $60 \%$ of the maximum growth rate is observed. This damping is because $\omega_{\text {Im }}$ is no longer zero but negative when $F_{h}>1$ because the bending disturbances have a viscous critical layer (Billant 2010). This is confirmed in figure 11: the corresponding 
(a)

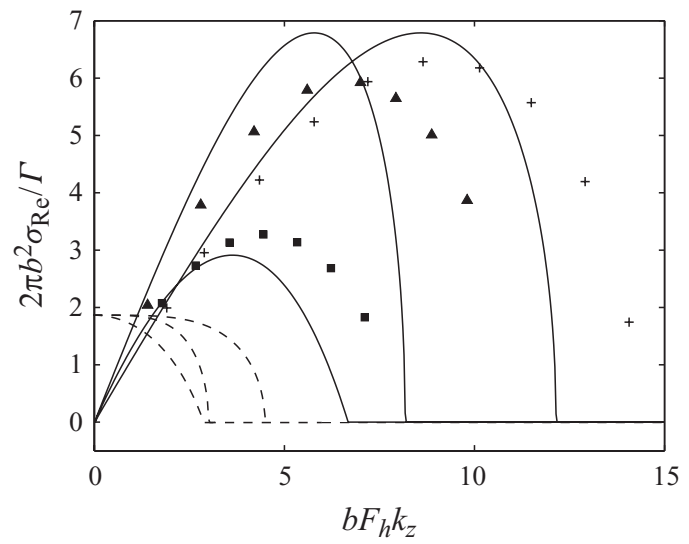

(b)

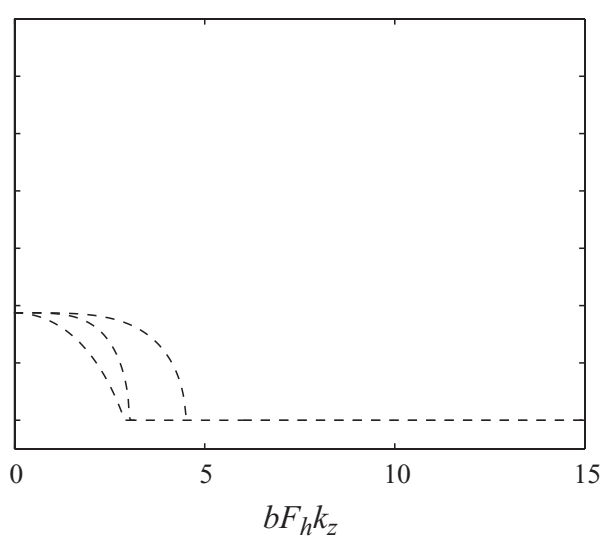

FIGURE 10. Similar to figure 2 but for various Froude numbers. (a) Symmetric mode and (b) antisymmetric mode. We recall that two row-wise periodicities are displayed: $\mu=\infty$ (solid lines) and $\mu=2$ (dashed lines). The curves represent the asymptotic theory and correspond, from right to left, to the Froude numbers: $F_{h}=0.1, F_{h}=1$ and $F_{h}=1.2$. The symbols correspond to the numerical results obtained for the dominant mode and for the same Froude numbers:,$+ F_{h}=0.1 ; \boldsymbol{\Lambda}, F_{h}=1 ; \mathbf{\square}, F_{h}=1.2$.

(a)

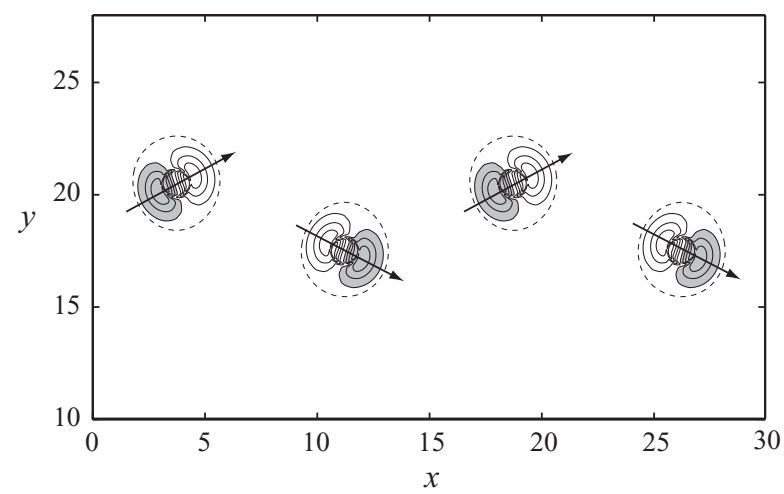

(b)

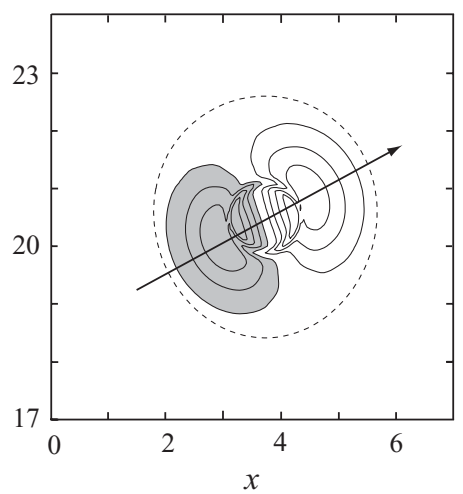

Figure 11. Similar to figure 3 but for $F_{h}=1.2$. Only numerical results are presented. $(a)$ The global view of the eigenmode and $(b)$ a close-up view of one vortex core showing the viscous critical layer.

numerical eigenmode exhibits a viscous critical layer in each vortex core at the radius where the angular velocity of the vortex is equal to the Brunt-Väisälä frequency. In contrast, the maximum growth rate of the two-dimensional pairing instabilities is not affected by the stratification (figure 10). Only the width of the unstable wavenumber band varies with $F_{h}$.

When $F_{h}$ further increases beyond 1.2 (figure 12a), the maximum growth rate of the zigzag instability (solid line) drops to zero at $F_{h}=1.83$ and then re-increases. The two-dimensional pairing instability therefore becomes dominant for $1.34<F_{h}<3.25$ since its maximum growth rate (dashed line) is independent of $F_{h}$. It should be stressed that these thresholds for the Froude number are specific to the spacing ratio $\kappa=0.2$ presented here and differ for other values of $\kappa$. The corresponding most amplified wavenumber of the three-dimensional instability (figure 12b) also drops to zero for 

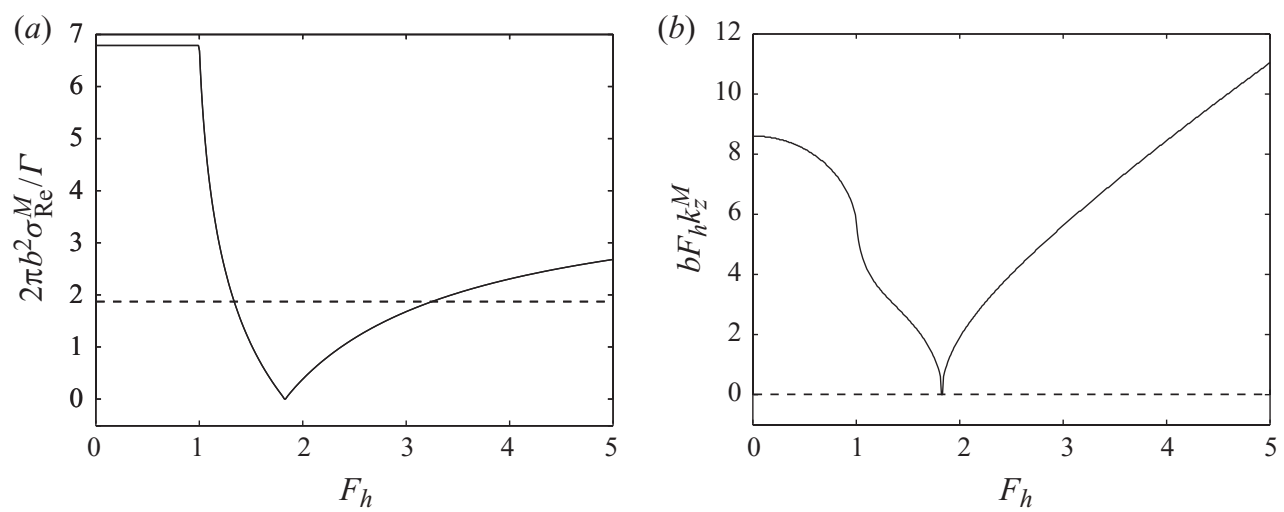

Figure 12. (a) Non-dimensional maximum growth rate $2 \pi b^{2} \sigma_{\mathrm{Re}}^{M} / \Gamma$ of the most unstable mode for $\mu=\infty$ (solid line) and $\mu=2$ (dashed line) and $(b)$ the corresponding unstable wavenumber $b F_{h} k_{z}^{M}$ as a function of the Froude number $F_{h}$ for the Kármán vortex street with close rows $(\kappa=0.2)$ in a non-rotating fluid.

(a)

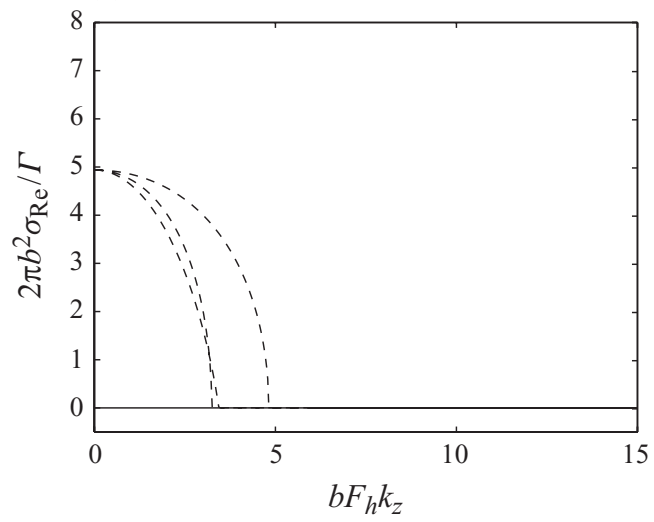

(b)

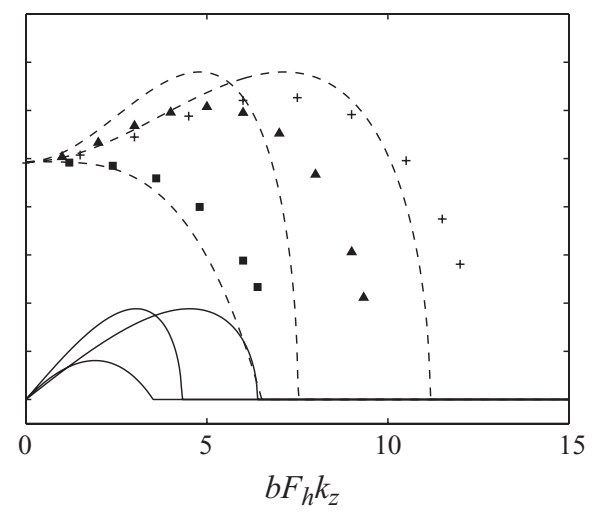

FIgURE 13. Similar to figure 10 but for the symmetric double row with $\kappa=0.5$. (a) Symmetric mode and (b) antisymmetric mode.

$F_{h}=1.83$ and then re-increases. In fact, the instability becomes antisymmetric for $F_{h}>1.83$, like in homogeneous fluids. This reversal of the symmetry is because the real part of the self-induction function (3.5) becomes negative.

However, it should be recalled that the asymptotic theory is restricted to moderate Froude numbers, $F_{h} \ll(b / a)^{2}$ (see $\S 3.1$ ). Therefore, the results found by Robinson \& Saffman (1982) in homogeneous fluid $\left(F_{h}=\infty\right)$ cannot be recovered by the present theory.

\subsection{Symmetric double row}

As seen in figure 13, the maximum theoretical growth rate for the symmetric double row also remains identical when the Froude number is increased from $F_{h}=0.1$ to $F_{h}=1$. However, when the Froude number is further increased to $F_{h}=1.2$, the $\mu=2$ instability is no longer most unstable for a finite value of $k_{z}$ because the threedimensional bending disturbances are strongly damped by a viscous critical layer as for the Kármán vortex street. The dominant instability is then the two-dimensional pairing mode. Good agreement is again found between numerical (symbols) and theoretical (dashed lines) results for the dominant mode in figure 13(b). 

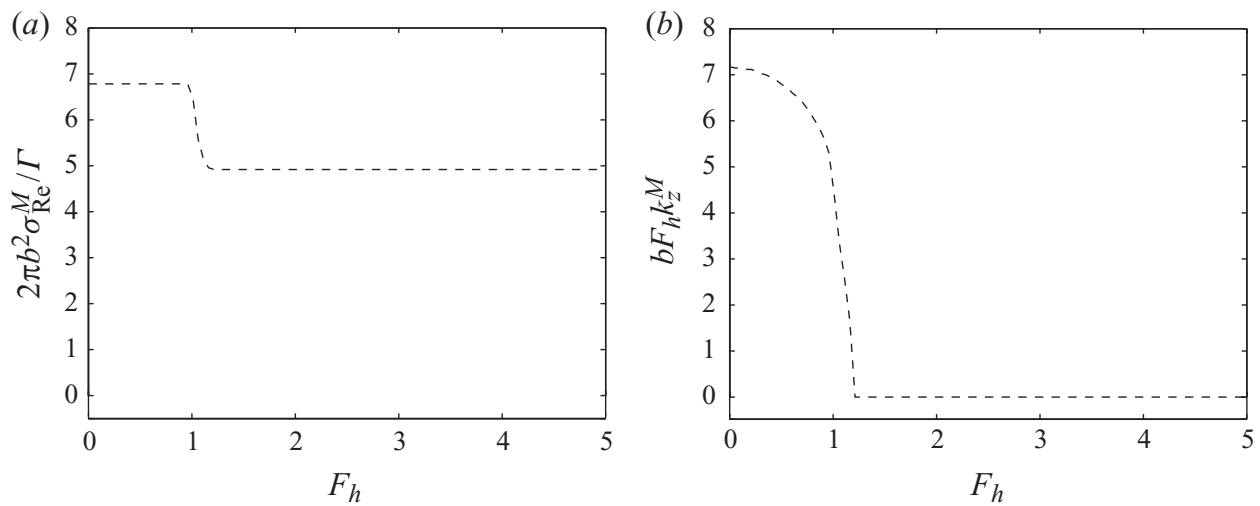

Figure 14. Similar to figure 12 but for the symmetric double row $(\kappa=0.5)$. (a) Symmetric mode and $(b)$ antisymmetric mode. Only the curves for $\mu=2$ (dominant instability) have been plotted.

Figure 14 shows the maximum growth rate and the most amplified wavenumber as a function of $F_{h}$. It confirms that the instability is three-dimensional only for $F_{h}<1.2$.

\subsection{Single row}

As discussed in $\S 5.3$, the dominant instability of the single row is a two-dimensional pairing instability. The stratification has thus no effect except that the width of the band of unstable vertical wavenumbers scales like $1 / F_{h}$ for $F_{h} \rightarrow 0$ and narrows when $F_{h}$ is increased.

\section{Strongly stratified and rotating fluid: effect of the Rossby number}

We now turn our attention to the effect of the Rossby number for a fixed Froude number. In the case of a rotating fluid $(R o \neq \infty)$, symmetric and antisymmetric modes do not decouple and the asymptotic eigenvalue problem (3.12) is solved numerically using the eigensolver eig of Matlab.

\subsection{Kármán vortex street}

We have determined the dominant mode of the Kármán vortex street for a strongly stratified fluid $\left(F_{h}=0.1\right)$ for Rossby numbers from non-rotating fluid $(R o \rightarrow \infty)$ to strong rotation $(R o=0.75)$ and for various spacing ratios. Only positive Rossby numbers need to be considered since the problem is independent of the sign of $R o$ because the vortices of the two rows are counter-rotating. In the rotating case, the problem depends on the core radius to separation distance $a / b$ because of the logarithm term in (3.5) that does not vanish. Here, we have taken $a / b=0.067$ as in the numerical stability analyses (see table 1).

Figure 15 shows the growth rate of the dominant mode for the two typical configurations studied in the previous section: close rows $(\kappa=0.2)$ and distant rows $(\kappa=0.5)$. In both cases, the most unstable mode remains qualitatively the same as in the non-rotating case: it is a $\mu=\infty$ three-dimensional bending instability for close rows and a two-dimensional pairing instability for distant rows. For close rows (figure 15a), we see that the maximum growth rate of the three-dimensional mode decreases significantly $(\sim 40 \%)$ for $R o=6$ compared to $R o \rightarrow \infty$ and then remains approximately constant when $R o$ is further decreased. The corresponding most unstable vertical wavenumber decreases monotonically with $R o$. The agreement 
(a)

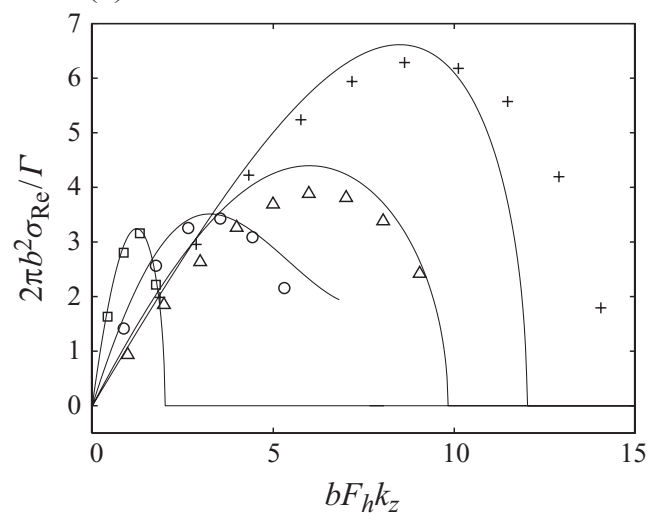

(b)

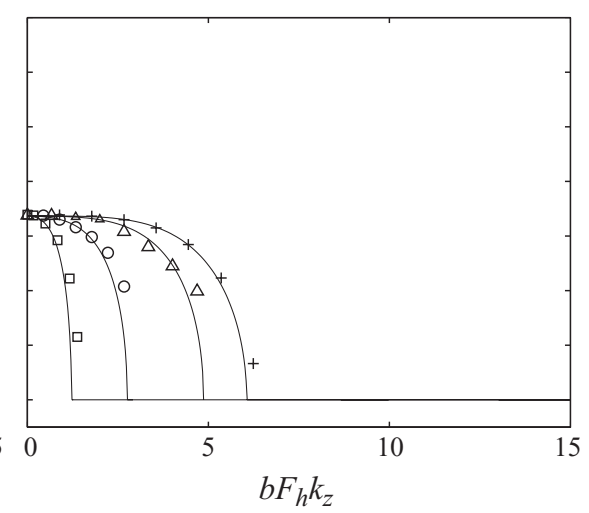

FIGURE 15. Non-dimensional growth rate $2 \pi b^{2} \sigma_{\mathrm{Re}} / \Gamma$ of the dominant mode as a function of the rescaled vertical wavenumber $b F_{h} k_{z}$ for $F_{h}=0.1$ for the Kármán vortex street with $(a)$ close rows $\kappa=0.2$ and $(b)$ distant rows $\kappa=0.5$. The dominant instability is the $\mu=\infty$ and $\mu=2$ mode, respectively. The solid lines correspond to the asymptotic theory and the symbols to the numerical results. In each plot, the curves from right to left correspond to the Rossby numbers:,$+ R o \rightarrow \infty ; \triangle, R o=6 ; \bigcirc, R o=2 ; \square, R o=0.75$. All curves have been determined for $a / b=0.067$.

(a)

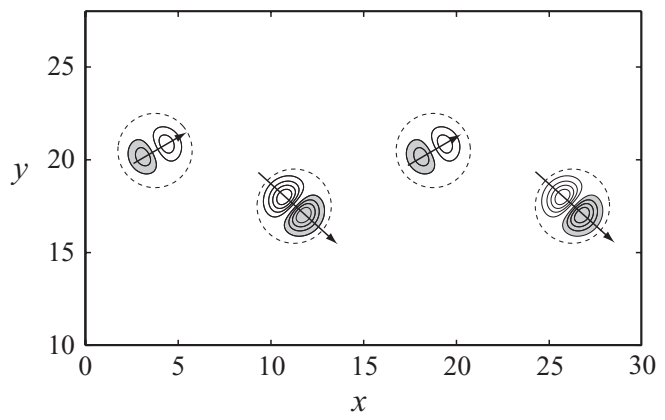

(b)

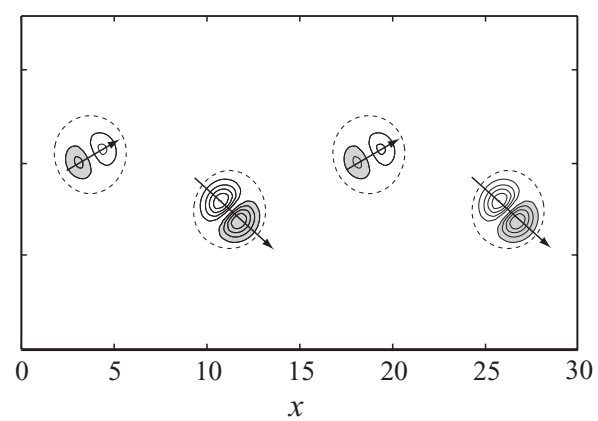

Figure 16. $(a, b)$ Similar to figure 3 but in a rotating fluid with $R o=2$.

between theoretical (solid lines) and numerical (symbols) results is again satisfactory. We further show in figure 16(a) the most amplified eigenmode for a typical rotating case $(R o=2)$. The eigenmode is not perfectly symmetric but only quasi-symmetric because symmetric and antisymmetric modes are coupled for finite Rossby numbers. The dipole perturbation structure is more intense for vortices in the lower row than in the upper row, indicating that anticyclonic vortices in the lower row are more displaced by the zigzag instability than the cyclonic vortices in the upper row. This is in agreement with the numerical results displayed in figure $16(b)$. For distant rows (figure $15 b$ ), we see that, although the maximum growth rate of the two-dimensional growth rate is not affected by rotation, the band of unstable vertical wavenumbers narrows dramatically when the rotation increases. Theoretical and numerical results are in excellent agreement.

Figure 17 further displays the maximum growth rate and most amplified wavenumber as a function of $R o$ for $\kappa=0.2$. We note that the most unstable vertical wavenumber decreases linearly to zero when $R o$ goes to zero. This behaviour is in 

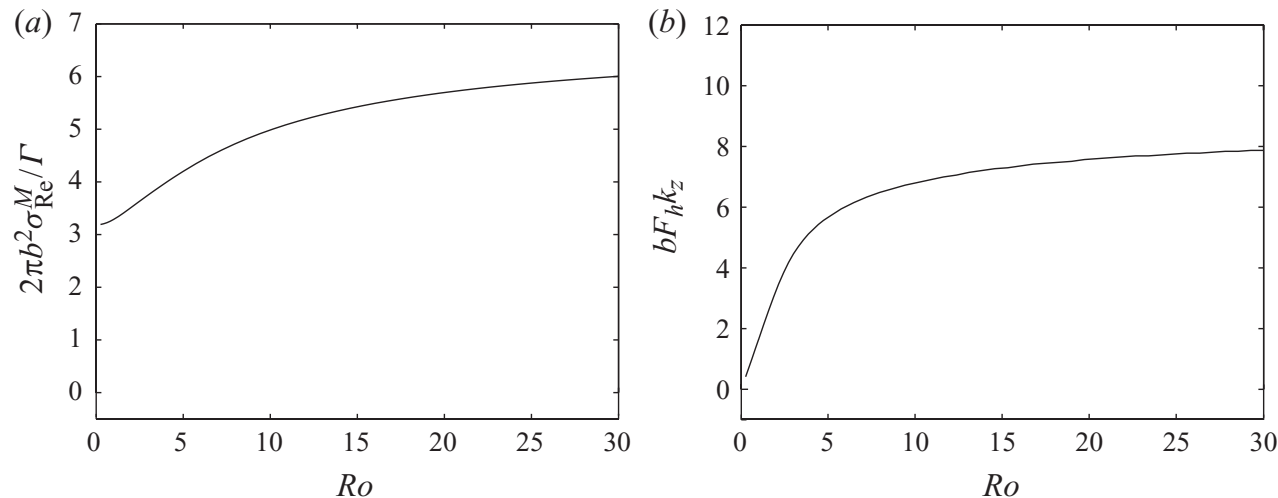

Figure 17. (a) Non-dimensional growth rate $2 \pi b^{2} \sigma_{\mathrm{Re}}^{M} / \Gamma$ of the most unstable mode and (b) the corresponding unstable wavenumber $b F_{h} k_{z}^{M}$ as a function of the Rossby number $R o$ for the Kármán vortex street with close rows $(\kappa=0.2)$ in a strongly stratified $\left(F_{h}=0.1\right)$ and rotating fluid.

agreement with the quasi-geostrophic scaling law and can be deduced directly from the asymptotic stability problem in the limit $R o \rightarrow 0$. Indeed, we see from (3.15) that the coefficients $A, B$ and $C$ are only functions of $b k_{z} F_{h} / R o$, through the arguments of the mutual induction functions $\psi$ and $\chi$. In addition, the self-induction terms $\left(b^{2} / a^{2}\right) \omega\left(k_{z}, F_{h}, \pm R o\right)$ that appear in the eigenvalue problem (3.12) converge to a single function when $R o \rightarrow 0$ :

$$
\lim _{R o \rightarrow 0} \frac{b^{2}}{a^{2}} \omega\left(k_{z}, F_{h}, \pm R o\right)=-\frac{b^{2} k_{z}^{2} F_{h}^{2}}{2 R o^{2}}\left(\ln \left(\frac{b k_{z} F_{h}}{2 R o}\right)+\ln \left(\frac{a}{b}\right)-\mathscr{D}\left(F_{h}\right)+\gamma_{e}\right),
$$

which is also a function of $b k_{z} F_{h} / R o$ for small $F_{h}$ since $\mathscr{D}\left(F_{h}\right) \approx$ const. for $F_{h}<0.5$ (see Appendix A and figure 22). Consequently, $k_{z}, F_{h}$ and $R o$ never appear separately but always as the group $b k_{z} F_{h} / R o$ in the eigenvalue problem (3.12). Thus, the growth rate is also only a function of $b k_{z} F_{h} / R o$, i.e. $\sigma\left(k_{z}, F_{h}, R o\right) \equiv \tilde{\sigma}\left(b k_{z} F_{h} / R o\right)$. It implies that the maximum vertical wavenumber of a three-dimensional instability scales like $R o /\left(b F_{h}\right)=N /\left(2 b \Omega_{b}\right)$ for small $R o$ and, similarly, that the size of the unstable band of vertical wavenumbers of the pairing instability scales as $R o /\left(b F_{h}\right)$ for small $R o$, as observed in figures 15 and 17.

\subsection{Symmetric double row}

Like for the Kármán vortex street, we have determined the dominant mode of the symmetric double row for $\kappa=0.5$ for various $R o$. Again, only positive Rossby numbers need to be considered because of the symmetry of the base flow. As seen in figure 18 the growth rate curves keep the same shape when $R o$ is decreased from $R o \rightarrow \infty$ to $R o=0.75$ but the unstable wavenumber band is narrowed. The most unstable mode remains a $\mu=2$ three-dimensional instability like in the non-rotating case. The agreement between asymptotic and numerical growth rates is again good especially for small wavenumbers. We see in figure 19 that the theoretical and numerical most amplified eigenmodes for $R o=2$ are also in very good agreement. The eigenmode with background rotation is similar to that for $R o \rightarrow \infty$ (figure 8) except that it is not exactly antisymmetric but only quasi-antisymmetric. Like for the Kármán vortex street, the anticyclonic vortices in the lower row are more displaced than the cyclonic vortices in the upper row. Figure 20 further shows the maximum growth rate and the 


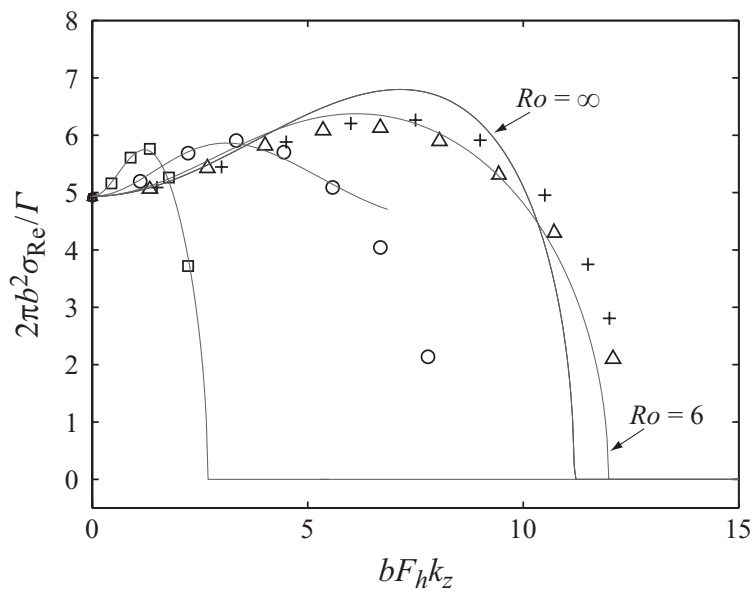

FIGURE 18. Similar to figure 15 but for the symmetric double row $(\kappa=0.5)$. We recall that four rotation rates are presented (from right to left curves):,$+ R o \rightarrow \infty ; \triangle, R o=6 ; \bigcirc, R o=2$; $\square, R o=0.75$. All curves have been determined for $a / b=0.067$.

(a)

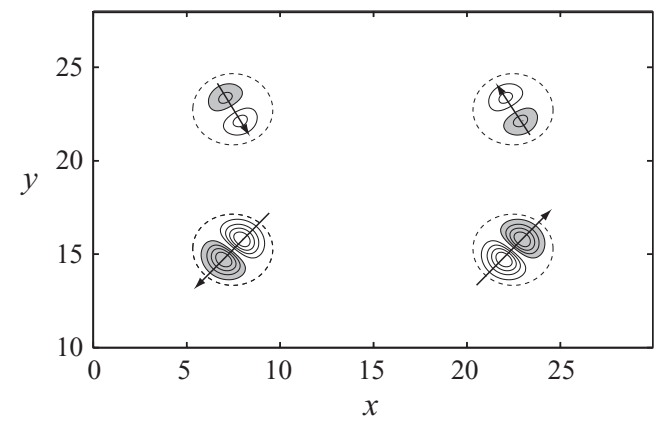

(b)

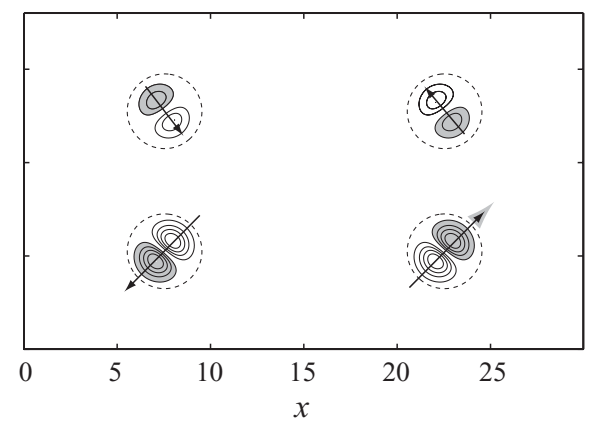

FIgURE 19. $(a, b)$ Similar to figure 8 but for a rotating fluid with $R o=2$.
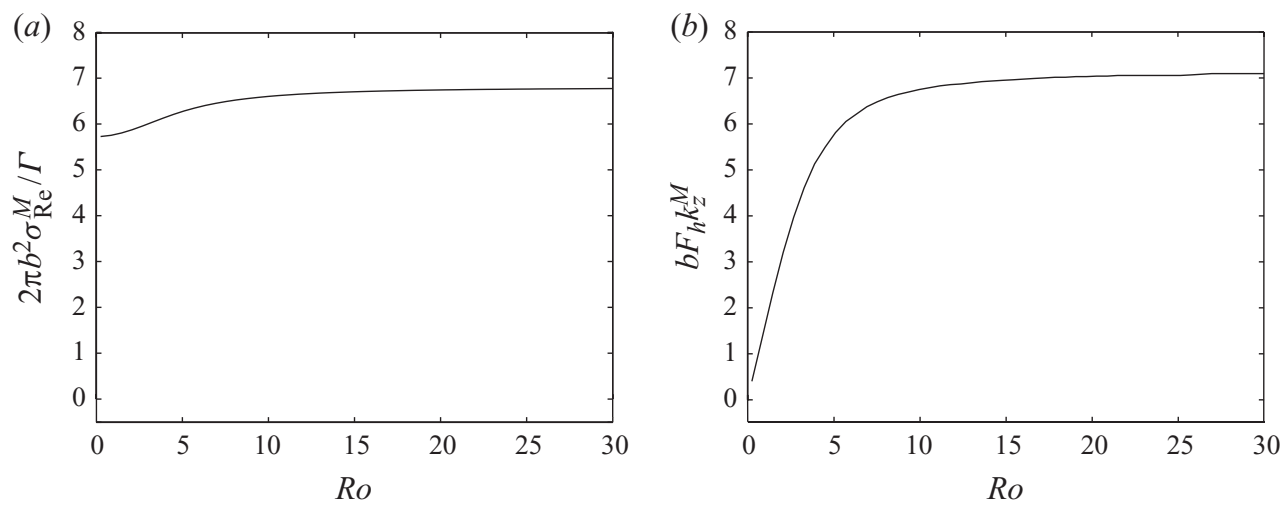

FIGURE 20. $(a, b)$ Similar to figure 17 but for the symmetric double row $(\kappa=0.5)$.

most unstable vertical wavenumber of the dominant mode as a function of the Rossby number Ro. Contrary to the Kármán vortex street, the maximum growth rate is only slightly reduced by the rotation: it is about $10 \%$ smaller for $R o \rightarrow 0$ compared to $R o \rightarrow \infty$. The most unstable vertical wavenumber is proportional to $R o$ for small $R o$ 


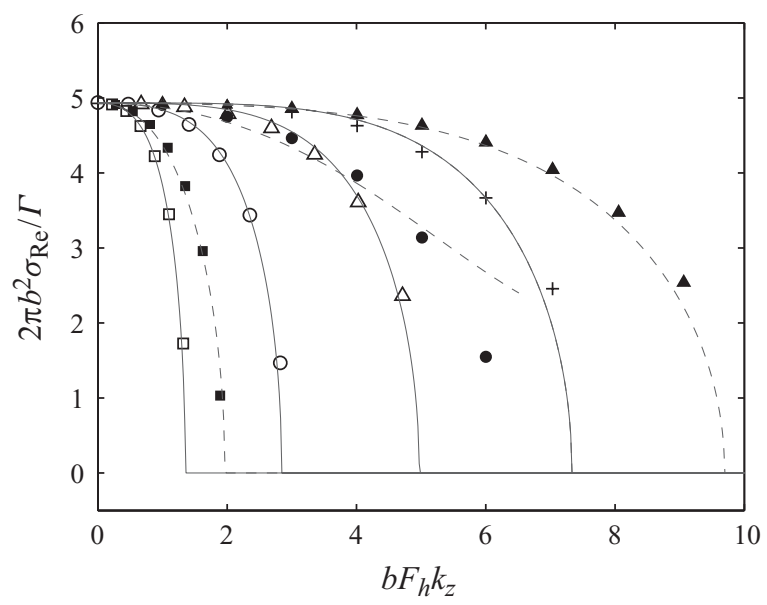

FIGURE 21. Non-dimensional growth rate $2 \pi b^{2} \sigma_{\mathrm{Re}} / \Gamma$ as a function of the rescaled vertical wavenumber $b F_{h} k_{z}$ for the single row in a strongly stratified $\left(F_{h}=0.1\right)$ fluid and for various Rossby numbers. The numerical results are represented by open symbols for positive Rossby numbers:,$+ R o \rightarrow \infty ; \triangle, R o=6 ; \bigcirc, R o=2 ; \square, R o=0.75$ and by filled symbols for negative Rossby numbers: $\boldsymbol{\Delta}, R o=-6 ; \boldsymbol{\bullet}, R o=-2 ; \mathbf{\square}, R o=-0.75$. The corresponding theoretical results are shown by solid lines for positive $R o$ and dashed lines for negative Ro. All curves have been determined for $a / b=0.067$.

like for the Kármán vortex street $(\S 7.1)$ and in agreement with the quasi-geostrophic theory.

\subsection{Single row}

The effect of rotation on the single row in a strongly stratified fluid is presented in figure 21. In this case, the velocity of the base flow is constant but in the opposite direction for $y \rightarrow \infty$ and $y \rightarrow-\infty$. Therefore, the base flow cannot be computed directly with a pseudo-spectral code that imposes periodic boundary conditions. To circumvent this difficulty, a symmetric double row with $\kappa=8$, i.e. with almost independent rows, has been first computed. Half of this flow, i.e. one row, has been cropped and the remaining part has been taken here as basic state. For this vortex array, the base flow is not symmetric so that the sign of the Rossby number matters. For positive Rossby numbers, the unstable band decreases monotonically when $R o$ decreases as observed before. For negative Rossby numbers, the unstable wavenumber band first widens for intermediate values of $R o$ but then also shrinks for small negative values. There is excellent agreement between numerical (symbols) and theoretical (solid lines) results except for $R o=-2$, where a departure is observed at large wavenumbers like for co-rotating vortex pairs (Billant et al. 2010).

\section{Conclusion}

We have investigated by asymptotic and numerical approaches the threedimensional stability in a stratified and rotating fluid of three steady vertical vortex arrays: the Kármán vortex street, the symmetric double row and the single row of co-rotating vortices. The asymptotic approach assumes long-wavelength bending perturbations and well-separated vortices. It is accurate to first order in the rescaled vertical wavenumber $k_{z}^{2} a^{2} F_{h}^{2}$ and in strain $\Gamma /\left(2 \pi b^{2}\right)$. The stability equations have the same form as those derived by Robinson \& Saffman (1982) in a homogeneous 
fluid. The differences between homogeneous and stratified and rotating fluids lie only in the explicit expression of the mutual induction functions and the self-induction function (Billant 2010).

Using this approach in a strongly stratified and non-rotating fluid and assuming a Lamb-Oseen vortex profile for each individual vortex, we have found that the Kármán vortex street for a spacing ratio $\kappa \leqslant 0.41$ and the symmetric double row for any spacing ratio are most unstable to a three-dimensional instability which bends the vortices. This bending instability is similar to the zigzag instability observed on vortex pairs in strongly stratified fluid (Billant \& Chomaz 2000; Otheguy et al. 2006b).

Similar bending instabilities also exist on vortex arrays in a homogeneous fluid (Robinson \& Saffman 1982) but a striking difference is the opposite symmetry of the unstable mode in a stratified fluid. For the Kármán vortex street, the most unstable mode for close rows is three-dimensional symmetric while it is three-dimensional antisymmetric in homogeneous fluid (Robinson \& Saffman 1982). Similarly, the symmetric double row is most unstable to a three-dimensional antisymmetric mode while it is a three-dimensional symmetric mode in a homogeneous fluid (Robinson \& Saffman 1982). This inversion of the symmetry of the unstable mode is rooted in the different sign of the self-induction in a stratified fluid compared to a homogeneous fluid (Billant et al. 2010).

Another important difference is the scaling of the most amplified vertical wavenumber: it scales like $1 /\left(b F_{h}\right)$ (or $1 /\left(h F_{h}\right)$ if $\kappa \ll 1$ for the symmetric double row) in a stratified fluid instead of $1 / b$ in a homogeneous fluid. The instabilities predicted in the present study are therefore expected to produce layers of typical thickness $b F_{h}$. In contrast, for both homogeneous and stratified fluids, the maximum growth rate scales like the strain $\Gamma /\left(2 \pi b^{2}\right)$. Interestingly, Praud et al. (2005) and Holford \& Linden (1999) have observed that the columnar vortices, created by towing a rake of vertical bars in a stratified fluid, are bent and evolve into pancake vortices. The present instability of the Kármán vortex street in a stratified fluid might explain these experimental observations.

It is also interesting to remark that the spacing ratio of atmospheric vortex streets in the wake of mountains or islands is typically 0.3-0.4 (Etling 1989, 1990; Li et al. 2000; Young \& Zawislak 2006), i.e. in the range unstable to the zigzag instability. However, an additional constraint for the zigzag instability to develop is that the vertical wavelength $\lambda$ should be smaller than the height $H$ of the vortices. This height $H$ is typically of the same order as the height of the islands or mountains, i.e. $H / D \approx 0.01-0.1$, where $D$ is the diameter of the mountain. The horizontal Froude number can be assumed to be small and the Rossby number large since the typical vortex radius is $O(20 \mathrm{~km})$ (Etling 1989). As seen in figure 6, the most amplified wavelength of the zigzag instability is then $\lambda \approx F_{h} b$ for $\kappa \approx 0.3$. Since the separation distance $h$ between the rows is approximately the same as the diameter of the mountain $D$, we obtain $\lambda / D \approx F_{h} / \kappa \approx 3 F_{h}$. For Froude numbers $F_{h} \approx 0.01-0.1$ typical of these flows, the ratios $\lambda / D$ and $H / D$ are therefore of the same order, meaning that at least one wavelength of the zigzag instability could fit in the vortex height. A similar discussion for the case of oceanic vortices can be found in Otheguy, Billant \& Chomaz (2006a).

For the Kármán vortex street with a large spacing ratio $\kappa>0.41$, or for the single row, the dominant instability in a strongly stratified fluid remains a two-dimensional pairing instability like in a homogeneous fluid. This result might seem in contradiction with the study of the nonlinear evolution of a horizontal shear layer in a stratified fluid by Basak \& Sarkar (2006), which reports the formation of a row of three-dimensional 
pancake vortices. However, Basak \& Sarkar (2006) have perturbed the initial twodimensional shear layer with relatively strong three-dimensional fluctuations. Such initial conditions should lead not only to the growth of the dominant mode but also to the growth of subdominant three-dimensional modes which are unstable over a large range of vertical wavenumbers in a strongly stratified fluid. Thus, the results of Basak \& Sarkar (2006) are not in contradiction with our finding that the single row is most unstable to two-dimensional disturbances.

Taking as a reference the stability properties of the three vortex arrays in a strongly stratified and non-rotating fluid, we have next investigated the effect of the Froude and Rossby numbers. In rescaled variables (non-dimensional growth rate $2 \pi b^{2} \sigma_{R e} / \Gamma$ and vertical wavenumber $b F_{h} k_{z}$ ), the Froude number has almost no effect as long as $F_{h}<1$. However, when $F_{h}$ is increased above unity, three-dimensional instabilities become strongly damped because long-wavelength bending perturbations exhibit a viscous critical layer.

For a strongly stratified fluid in the presence of background rotation, symmetric and antisymmetric modes do not decouple but we have always found that the dominant instability remains qualitatively similar to the non-rotating case: it is a three-dimensional zigzag instability for the Kármán vortex street with a small spacing ratio and for the symmetric double row with any spacing ratio; it is a two-dimensional pairing instability for the Kármán vortex street with a large spacing ratio and for the single row. However, the most unstable wavenumber of three-dimensional instabilities decreases with rotation and scales like $R o /\left(b F_{h}\right)=N /\left(2 b \Omega_{b}\right)$ for small $R o$. In this quasi-geostrophic limit, the decoupling between symmetric and antisymmetric modes is also recovered and the dominant three-dimensional instabilities have the same symmetry as for a stratified and non-rotating fluid.

The asymptotic results are in good agreement with the numerical stability analyses for all the Rossby and Froude numbers investigated even for finite wavelengths. In Appendix B, we also study numerically the effect of larger vortex radius to separation distance ratio, $a / b$, and show that the asymptotic theory remains qualitatively valid. This proves that the theory can be used to predict the stability of almost all vortex configurations. This should be useful since numerical stability analysis as performed here requires huge computing resources compared to the theory.

Finally, we emphasize that even if the theory is restricted to displacement perturbation of vortices, the results of the numerical stability analyses prove that these perturbations, either two-dimensional or three-dimensional, are the most unstable in stratified and rotating fluids over a wide range of Froude and Rossby numbers. We have not found any other dominant perturbations of a different nature, at least over the range of vertical wavenumbers investigated.

However, other types of instabilities could exist for higher vertical wavenumbers. For example, the centrifugal instability is known to exist when $R o<-1 / 2$ in the case of a Lamb-Oseen vortex. The maximum growth rate $\sigma_{c}$ of this instability is reached for $k_{z} \rightarrow \infty$ in the inviscid limit. In stratified and rotating fluids, $\sigma_{c}$ is independent of the stratification and given by $\sigma_{c}=\Gamma \sqrt{-\min (\phi)} /\left(2 \pi a^{2}\right)$, where $\min (\phi)$ is the minimum over $r$ of the generalized Rayleigh discriminant $\phi=\left(2 \Omega+R o^{-1}\right)\left(2 \Omega+r \mathrm{~d} \Omega / \mathrm{d} r+R o^{-1}\right)$ (Kloosterziel \& van Heijst 1991; Billant \& Gallaire 2005). When $R o$ increases from $R o=-\infty$, the maximum growth rate increases up to $\sigma_{\text {c max }}=0.3 \Gamma /\left(2 \pi a^{2}\right)$ at $R o=-1.6$ and then decreases back to zero at $R o=-1 / 2$. Therefore, the centrifugal instability can be stronger than the zigzag instability when $\sigma_{c \max }>\sigma=O\left(\Gamma /\left(2 \pi b^{2}\right)\right)$, i.e. typically when $a / b<O(1)$, in an intermediate band of Rossby numbers around $|R o|=1.6$ whose extent depends on the ratio $a / b$. 

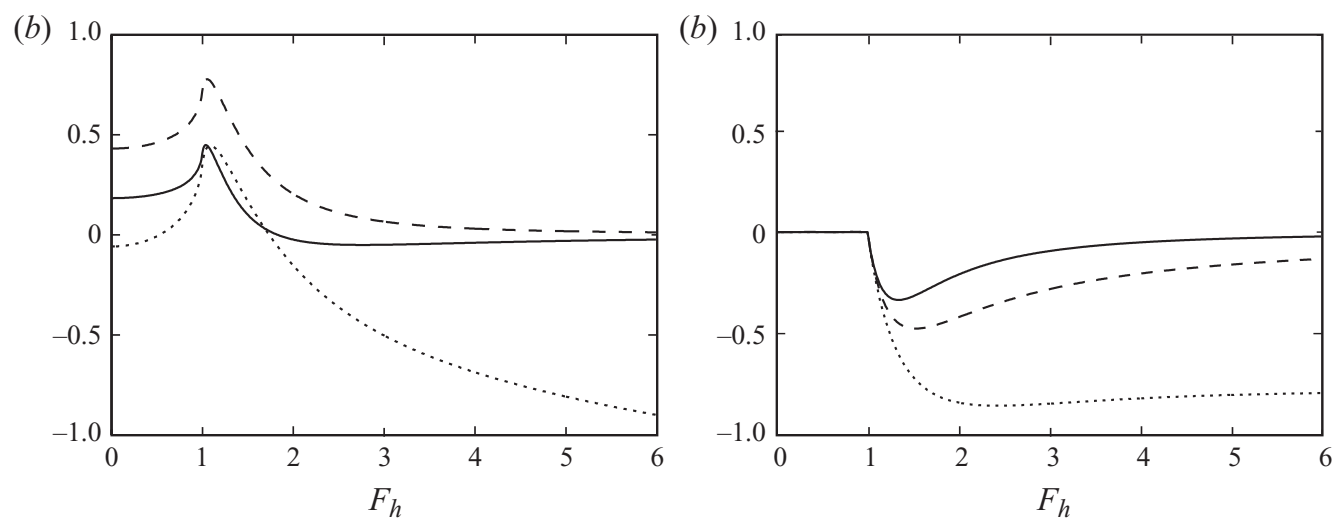

FIGURE 22. Real part $(a)$ and imaginary part $(b)$ of the parameters $\mathscr{A}$ (solid line), $\mathscr{B}$ (dashed-line) and $\mathscr{D}$ (dotted line) as a function of the Froude number $F_{h}$ for the Lamb-Oseen vortex.

\section{Appendix A. Expression of the parameters $\mathscr{A}, \mathscr{B}$ and $\mathscr{D}$}

The parameters $\mathscr{A}, \mathscr{B}$ and $\mathscr{D}$ used in the expression of the self-induction function $\omega$ valid in stratified and rotating fluids (see §3.1) depend on the Froude number and the non-dimensional angular velocity $\Omega$ of the individual vortices. Their expressions are (Billant 2010)

$$
\begin{aligned}
& \mathscr{A}\left(F_{h}\right)=\lim _{\eta_{0} \rightarrow \infty} \int_{0}^{\eta_{0}} \frac{r^{3} \Omega(r)^{4}}{1-F_{h}^{2} \Omega(r)^{2}} \mathrm{~d} r-\frac{1}{4 F_{h}^{2}} \ln \left(1-\frac{F_{h}^{2}}{\eta_{0}^{4}}\right), \\
& \mathscr{B}\left(F_{h}\right)=\lim _{\eta_{0} \rightarrow \infty} \int_{0}^{\eta_{0}} \frac{r^{3} \Omega(r)^{3}}{1-F_{h}^{2} \Omega(r)^{2}} \mathrm{~d} r-\frac{1}{4 F_{h}} \ln \left(\frac{\eta_{0}^{2}-F_{h}}{\eta_{0}^{2}+F_{h}}\right), \\
& \mathscr{D}\left(F_{h}\right)=\lim _{\eta_{0} \rightarrow \infty} \int_{0}^{\eta_{0}} \frac{r^{3} \Omega(r)^{2}}{1-F_{h}^{2} \Omega(r)^{2}} \mathrm{~d} r-\frac{1}{4} \ln \left(\eta_{0}^{4}-F_{h}^{2}\right),
\end{aligned}
$$

where $r$ is the radius non-dimensionalized by $a$. These parameters are plotted in figure 22 for the Lamb-Oseen vortex profile.

\section{Appendix B. Strongly stratified and non-rotating fluid: effect of the vortex radius to separation distance ratio: $a / b$}

In this appendix, we investigate the effect of the vortex radius to separation distance ratio $a / b$ for the Kármán vortex street with $\kappa=0.2$ for a fixed set of Froude and Rossby numbers: $F_{h}=0.1$ and $R o \rightarrow \infty$. Figure 23 shows the growth rate of the dominant mode for various ratios $a / b$ from well-separated vortices $(a / b=0.067)$ to very close vortices $(a / b=0.133)$. These cases correspond to the basic states labelled $\mathrm{K} 0.2, \mathrm{~K} 0.2 \mathrm{~b} 10$ and $\mathrm{K} 0.2 \mathrm{~b} 7.5$ in table 1 . We recall that the effective ratio $a / d$, where $d$ is the minimum distance between vortices is even larger and goes here from $a / d=0.124$ to $a / d=0.248$. As the vortices get closer, the maximum growth rate decreases slightly and the band of unstable vertical wavenumbers widens. We see a departure between the numerical results (dotted line with symbols) and the asymptotic theory (solid line) which is valid only for well-separated vortices (i.e. at leading order in $a / b$ ). However, the instability remains qualitatively the same. 


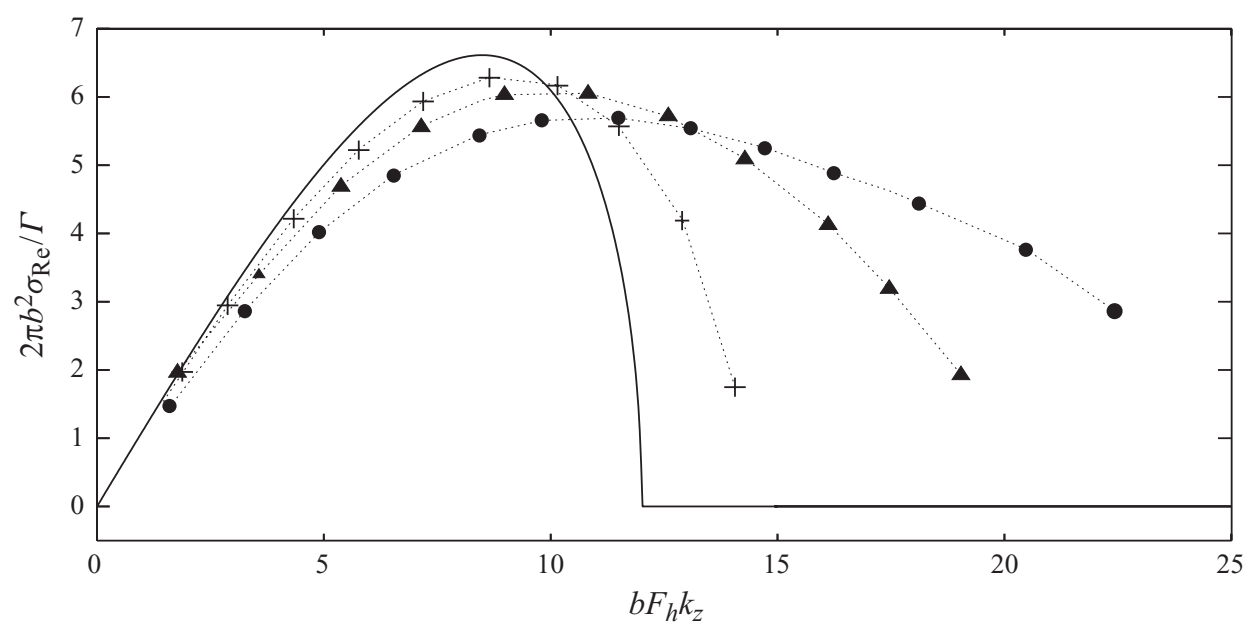

FIGURE 23. Non-dimensional growth rate $2 \pi b^{2} \sigma_{\mathrm{Re}} / \Gamma$ of the dominant mode as a function of the rescaled vertical wavenumber $b F_{h} k_{z}$ with $F_{h}=0.1$ and $R o \rightarrow \infty$ for the Kármán vortex street with $\kappa=0.2$. The dotted lines with symbols correspond to numerical results and the solid line to the asymptotic theory valid for well-separated vortices. Different vortex radius to separation distance ratios are presented:,$+ a / b=0.067 ; \mathbf{\Lambda}, a / b=0.100 ; \bullet, a / b=0.133$.

\section{REFERENCES}

BASAK, S. \& SARKar, S. 2006 Dynamics of a stratified shear layer with horizontal shear. J. Fluid Mech. 568, 19-54.

Billant, P. 2010 Zigzag instability of vortex pairs in stratified and rotating fluids. Part 1. General stability equations. J. Fluid Mech. 660, 354-395.

Billant, P. \& Chomaz, J.-M. 2000 Experimental evidence for a new instability of a vertical columnar vortex pair in a strongly stratified fluid. J. Fluid Mech. 418, 167-188.

Billant, P., Deloncle, A., Chomaz, J.-M. \& Otheguy, P. 2010 Zigzag instability of vortex pairs in stratified and rotating fluids. Part 2. Analytical and numerical analyses. J. Fluid Mech. 660, 396-429.

Billant, P. \& Gallaire, F. 2005 Generalized Rayleigh criterion for non-axisymmetric centrifugal instabilities. J. Fluid Mech. 542, 365-379.

Crow, S. C. 1970 Stability theory for a pair of trailing vortices. AIAA J. 8, 2172-2179.

Deloncle, A., Chomaz, J.-M. \& Billant, P. 2007 Three-dimensional stability of a horizontally sheared flow in a stably stratified fluid. J. Fluid Mech. 570, 297-305.

EtLing, D. 1989 On atmospheric vortex streets in the wake of large islands. Meteorol. Atmos. Phys. 41, 157-164.

EtLing, D. 1990 Mesoscale vortex shedding from large islands: a comparison with laboratory experiments of rotating stratified flows. Meteorol. Atmos. Phys. 43, 145-151.

Holford, M. \& Linden, P. F. 1999 Turbulent mixing in a stratified fluid. Dyn. Atmos. Oceans 30, $173-198$

JimeneZ, J. 1975 Stability of a pair of co-rotating vortices. Phys. Fluids 18, 1580-1581.

VON KÁRMÁN, T. 1911 Über den mechanismus des widerstands, den ein bewegter körper in einer flüssigkeit erfährt. Göttinger Nachr., Math. Phys. Kl. pp. 509-517.

vON KÁRMÁN, T. 1912 Über den mechanismus des widerstands, den ein bewegter körper in einer flüssigkeit erfährt. Göttinger Nachr., Math. Phys. Kl. pp. 547-556.

vON KÁRMÁN, T. \& RuBACH, H. L. 1912 Über den mechanismus des flüssigkeits- und luftwiderstands. Phys. Z 13, 49-59.

Kloosterziel, R. C. \& van Heisst, G. J. F. 1991 An experimental study of unstable barotropic vortices in a rotating fluid. J. Fluid Mech. 223, 1-24.

Lamb, H. 1932 Hydrodynamics. Cambridge University Press. 
Leblanc, S. \& Cambon, C. 1998 Effects of the Coriolis force on the stability of Stuart's vortices. J. Fluid Mech. 356, 353-379.

Li, X., Clemente-Colón, P., Pichel, W. G. \& Vachon, P. W. 2000 Atmospheric vortex streets on a RADARSAT SAR image. Geophys. Res. Lett. 27 (11), 1655-1658.

Otheguy, P., Billant, P. \& Chomaz, J.-M. 2006a The effect of planetary rotation on the zigzag instability of co-rotating vortices in a stratified fluid. J. Fluid Mech. 553, 273-281.

Otheguy, P., Chomaz, J.-M. \& Billant, P. $2006 b$ Elliptic and zigzag instabilities on co-rotating vertical vortices in a stratified fluid. J. Fluid Mech. 553, 253-272.

Potylitsin, P. G. \& Peltier, W. R. 1998 Stratification effects on the stability of columnar vortices on the $f$-plane. J. Fluid Mech. 355, 45-79.

Potylitsin, P. G. \& Peltier, W. R. 1999 Three-dimensional destabilization of Stuart vortices: the influence of rotation and ellipticity. J. Fluid Mech. 387, 205-226.

Praud, O., Fincham, A. M. \& Sommeria, J. 2005 Decaying grid turbulence in a strongly stratified fluid. J. Fluid Mech. 522, 1-33.

Robinson, A. C. \& Saffman, P. G. 1982 Three-dimensional stability of vortex arrays. J. Fluid Mech. 125, 411-427.

VInCENT, A. \& MenEguzzi, M. 1991 The spatial structure and statistical properties of homogeneous turbulence. J. Fluid Mech. 225, 1-20.

Young, G. S. \& ZawislaK, J. 2006 An observational study of vortex spacing in island wake vortex streets. Mon. Weath. Rev. 134 (8), 2285-2294. 